\title{
Geometry and Kinematics of Two Skyrmions
}

\author{
M. F. Atiyah ${ }^{1}$ and N.S. Manton ${ }^{2}$ \\ 1 Trinity College, Cambridge CB2 1TQ, UK \\ 2 Department of Applied Mathematics and Theoretical Physics, Silver Street, Cambridge CB3 9EW, \\ UK
}

Received June 15, 1992; in revised form October 13, 1992

\begin{abstract}
In Skyrme's soliton model of baryons, a single Skyrmion has six degrees of freedom, so it is expected that two-Skyrmion dynamics at modest energies can be modelled by motion on a 12-dimensional space of Skyrme fields. A candidate for this space is generated by the gradient flow of the potential energy function, descending from the unstable, baryon number two, hedgehog solutions of the Skyrme field equation. An apparently very similar space is obtained by restricting the gradient flow to the Skyrme fields derived from $S U(2)$ Yang-Mills instantons of charge two. On both of these spaces, one may quotient out by the group of translations and isospin rotations. Hartshorne's geometrical description of charge two instantons leads us to a conjecture for the global structure of the 6-dimensional quotient space. The conjectured structure is that of complex projective 3 -space, with complex conjugate points on one projective plane identified and the real points in this plane removed.
\end{abstract}

\section{Introduction}

A fundamental problem in non-integrable soliton models of elementary particles is to find an approximate description of soliton interactions in which each soliton has the same number of degrees of freedom as a single isolated soliton. Such a description reduces a field theory with infinitely many degrees of freedom to a "particle" theory, and is appropriate at modest energies where radiation and particle-antiparticle pair production can be neglected. In certain special Lagrangian field theories, there is a moduli space of exact static solutions of a Bogomol'nyi-type equation [1], representing multi-soliton configurations, and the dimension of the $k$-soliton moduli space, suitably defined, is of the form $N k$, where $N$ is the number of degrees of freedom of a single soliton. For $S U$ (2) Yang-Mills-Higgs monopoles, for example, $N$ is four [2], and for vortices in the abelian Higgs model, $N$ is two [3]. The moduli space is a flat valley of minimal energy in the configuration space of fields at a given time, and has a natural Riemannian metric inherited from the kinetic terms of the field theory Lagrangian. The classical dynamics of the solitons is modelled by geodesic motion on the moduli 
space [4], and quantized dynamics may be approximated by quantizing the moduli, with a purely kinetic Hamiltonian proportional to the Laplace-Beltrami operator [5].

In more general field theories, with solitons but no Bogomol'nyi equation, a substitute for the moduli space must be found. One again seeks a valley in the field configuration space, as flat as possible, and of the appropriate dimension. The fields of minimal energy, for given soliton number, need to be included but usually do not have sufficient dimensions. One proposal, suggested by analogies with molecular reaction dynamics, is to use the gradient flow curves, or paths of steepest descent, on the field configuration space [6]. In field theory, the gradient flow curves are solutions of a generalized diffusion equation which can be derived from the Lagrangian. They are tangent at their upper ends to the unstable modes of a static, saddle point solution of the field equations, and generically they descend to minimal energy solutions. The moduli space $M$ is defined as the union of gradient flow curves descending from a suitable family of saddle points, completed, if necessary, by gradient flow curves descending from lower saddle points.

Ideally, $M$ should be a manifold, but it may have singularities. $M$ inherits from the Lagrangian of the field theory a metric and potential energy function, so that there is a well-defined Lagrangian dynamics on $M$. Motion on $M$ will approximate the field theory dynamics of the solitons provided the potential on $M$ is not very steep and velocities remain modest, so that soliton interactions do not excite much radiation. Quantized soliton dynamics is now modelled by a Hamiltonian on $M$ which has a potential term in addition to the previous purely kinetic term.

The Skyrme model is an example of a field theory with solitons, but no Bogomol'nyi equation [7]. Skyrme fields, at a given time, are characterized by an integer topological invariant, which may be identified both as the soliton number and physically as the baryon number. This integer is conserved classically, and also, presumably, quantum mechanically. The soliton has a spherically symmetric "hedgehog" form, and is known as a Skyrmion. The Skyrmion solutions of the Skyrme field equation have six degrees of freedom - three translational and three rotational. After quantization of these degrees of freedom, the Skyrmion models a spin $1 / 2$, isospin $1 / 2$ baryon - that is, a proton or neutron [8].

The problem of interest here is two-Skyrmion interactions. We therefore seek a 12-dimensional moduli space of Skyrme fields of baryon number $B=2$. The known static solutions of the Skyrme equation in the $B=2$ sector are the toroids [9], which are stable, and the $B=2$ hedgehogs [10], which have higher energy and are unstable. We shall assume that there are no other genuine solutions with energy less than that of the hedgehogs. However, there are the virtual solutions consisting of two infinitely separated Skyrmions, whose energy is less than that of the hedgehogs but greater than that of the toroids. The family of $B=2$ hedgehog solutions is 6-dimensional, and linear analysis shows that each hedgehog solution has six unstable modes [11], so the union of gradient flow curves descending from the family of solutions is 12 dimensional. The generic curve descends to one of the toroids, but some special curves descend to infinitely separated Skyrmions. This union of gradient flow curves, completed with the curves that descend from infinitely separated Skyrmions to a toroid, is our first candidate for the two-Skyrmion moduli space. We denote it by $M_{12}$. A sketch of it is shown in Fig. 1 .

There have been attempts to model two-Skyrmion interactions using fewer than twelve degrees of freedom. Quantizing the eight degrees of freedom of the toroids gives a qualitatively sensible description of the two-baryon bound state, the deuteron, but the binding is much too strong, and clearly there are insufficient degrees of 


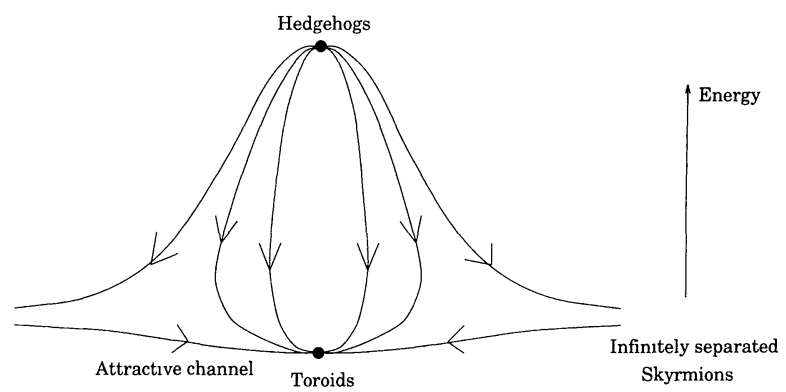

Fig. 1. Sketch of the gradient flow curves on the two-Skyrmion moduli space $M_{12}$

freedom for the two baryons to separate [12]. The union of gradient flow curves descending from infinitely separated Skyrmions to the toroids is a 10-dimensional manifold, called the "attractive channel". Approximations to the attractive channel have been determined in various ways, and the degrees of freedom occurring there have been (approximately) quantized. The result is a better description of the deuteron, but it is still quantitatively poor. The problem with the attractive channel is that the relative orientation of the two Skyrmions is partially frozen. This is reasonable on energetic grounds when the Skyrmions are close together, but when the Skyrmions are well separated they should be free to rotate independently. Nevertheless, the attractive channel fields are of great importance in all low-energy two-Skyrmion phenomena.

There is an independent way of reducing the degrees of freedom of Skyrme field theory. Recently, it was observed that a Skyrme field in $\mathbb{R}^{3}$ may be obtained from an $S U(2)$ Yang-Mills instanton in $\mathbb{R}^{4}$ by calculating the holonomy of the instanton along all lines parallel to the Euclidean time axis [13]. A dynamical Skyrme field is obtained when the instanton parameters depend on the physical time. The charge $k$ instantons give rise to an $8 k$-1-dimensional manifold of baryon number $k$ Skyrme fields. These instanton-generated Skyrme fields do not satisfy the static Skyrme equation, but for suitable parameter values they apparently give good approximations to most known Skyrmion and multi-Skyrmion configurations. They capture the positional and orientational degrees of freedom of separated Skyrmions, and have some degrees of freedom to spare (including a scale factor for each Skyrmion). They fail to model the radiation modes of the Skyrme field, but this does not matter for our purposes.

The manifold of $B=2$ Skyrme fields generated by instantons of charge two is 15-dimensional, and we denote it by $\widetilde{M}_{15}$. The metric and potential energy function on $\widetilde{M}_{15}$, inherited from the Skyrme Lagrangian, define a physically reasonable Lagrangian dynamics for these fields. (This is rather a hybrid construction, as the instantons are already defined dynamically as solutions of the Yang-Mills self-dual equations.) The fields of minimal potential energy are close to the toroid solutions of the Skyrme equation, with an energy less than $2 \%$ higher. The $B=2$ hedgehog fields of minimal energy in $\widetilde{M}_{15}$ correspond to the $B=2$ hedgehog solutions of the Skyrme equation. Within $\widetilde{M}_{15}$, they have six degrees of freedom, and they appear to be unstable. This has not been checked numerically, but there are six directions in $\widetilde{M}_{15}$ which correspond qualitatively and semi-quantitatively to the unstable modes of the hedgehog solutions. We shall assume that there are indeed six unstable modes. Then the gradient flow curves descending from these minimal energy $B=2$ hedgehogs, derived using the metric and potential on $\widetilde{M}_{15}$, generate a 12-dimensional space. The completion of the 
union of gradient flow curves, which we denote by $\widetilde{M}_{12}$, is our second candidate for the two-Skyrmion moduli space. One may hope that $\widetilde{M}_{12}$ is uniformly close to $M_{12}$ in the full Skyrme field configuration space.

By exploiting the geometry of the manifold of charge two instantons, we can visualize $\widetilde{M}_{12}$ somewhat more clearly than $M_{12}$. Our main result is a conjecture for the global structure of the quotient moduli space $\widetilde{M}_{12} /\left(\mathbb{R}^{3} \times S O(3)_{\text {isospin }}\right)$, where $\mathbb{R}^{3} \times S O(3)_{\text {isospin }}$ is the freely acting group of translations and isospin rotations. The conjecture is that this quotient space is diffeomorphic to $\mathbb{C} P_{3}$, with complex conjugate points on a $\mathbb{C} P_{2}$ subspace identified, and with the $\mathbb{R} P_{2}$ of real points in this subspace removed. There remains a major computational challenge to construct $M_{12}$ and $\widetilde{M}_{12}$ in detail, and to verify that they are close to each other, and so diffeomorphic. It will then be necessary to calculate the metrics and potential energy functions on them, in order to calculate two-Skyrmion bound and scattering states. The problems seem formidable, but the analogous programme for two-monopole interactions, where there are fewer degrees of freedom, is well-advanced $[4,5]$.

In Sect. 2 we summarize relevant material about Skyrme fields and Skyrmions. In Sect. 3 we discuss the Skyrme fields generated from instantons, and in Sect. 4 the geometry of charge two instantons, developing the material in [13]. Section 5 is concerned with the $B=2$ Skyrme fields of hedgehog type generated by instantons. In Sect. 6 the stability properties of hedgehog fields are discussed. In Sect. 7 we describe the low energy attractive channel for two Skyrmions, and its relation to the moduli space of two Bogomol'nyi monopoles. Finally, in Sect. 8 we explain and discuss our conjecture for the global structure of the quotient moduli space for two Skyrmions.

\section{Skyrmions}

A Skyrme field is a smooth, scalar $S U(2)$-valued field $U\left(x_{0}, \mathbf{x}\right)$, defined in one time and three space dimensions. $U$ may be expanded as

$$
U\left(x_{0}, \mathbf{x}\right)=\sigma\left(x_{0}, \mathbf{x}\right)+i \pi\left(x_{0}, \mathbf{x}\right) \cdot \tau,
$$

where $\tau_{1}, \tau_{2}$ and $\tau_{3}$ are Pauli matrices. $\pi_{1}, \pi_{2}$ and $\pi_{3}$ are a triplet of pion fields, and $\sigma$ is the "sigma" field, constrained by

$$
\sigma^{2}+\pi_{1}^{2}+\pi_{2}^{2}+\pi_{3}^{2}=1 .
$$

There is a Lie algebra valued current $L_{\mu}=U^{-1} \partial_{\mu} U(\mu=0,1,2,3)$ in terms of which the Lagrangian density is [7]

$$
\mathscr{D}=-\frac{1}{2} \operatorname{Tr}\left(L_{\mu} L^{\mu}\right)+\frac{1}{16} \operatorname{Tr}\left(\left[L_{\mu}, L_{\nu}\right]\left[L^{\mu}, L^{\nu}\right]\right) .
$$

Here, indices are raised using the Minkowski metric $(+,-,-,-)$ and $[$,$] denotes the$ bracket of the $S U(2)$ Lie algebra. Natural length and energy units have been chosen.

The first term in $\mathscr{B}$ is the "sigma model" Lagrangian, and the second is the "Skyrme term", introduced by Skyrme to ensure the existence of a static soliton solution of the field equation. Various modifications and replacements for the Skyrme term have been proposed, but none leads to a clearly superior model, so we shall not discuss them. According to (2.3) the pions are massless Goldstone bosons. It is straightforward to add a pion mass term to (2.3), but the symmetry of the model is 
then reduced. The symmetry group of the Lagrangian density (2.3) is the Poincare group, together with chiral rotations

$$
U \rightarrow U_{L} U U_{R}
$$

where $U_{L}$ and $U_{R}$ are constant $S U(2)$ matrices.

The potential energy of a Skyrme field at a given time is

$$
V=\int d^{3} x\left\{-\frac{1}{2} \operatorname{Tr}\left(L_{i} L_{\imath}\right)-\frac{1}{16} \operatorname{Tr}\left(\left[L_{i}, L_{j}\right]\left[L_{i}, L_{j}\right]\right)\right\}
$$

$(i, j=1,2,3)$. Its kinetic energy is

$$
T=\int d^{3} x\left\{-\frac{1}{2} \operatorname{Tr}\left(L_{0} L_{0}\right)-\frac{1}{8} \operatorname{Tr}\left(\left[L_{0}, L_{i}\right]\left[L_{0}, L_{i}\right]\right)\right\},
$$

which is an expression quadratic in time-derivatives. A Skyrme field with finite potential energy tends to a constant value at infinity, independent of direction. If the field also has finite kinetic energy, then this constant cannot change with time. It is therefore conventional to suppose that a physical Skyrme field satisfies the boundary condition

$$
U\left(x_{0}, \mathbf{x}\right) \rightarrow 1
$$

as $|\mathbf{x}| \rightarrow \infty$. This boundary condition implies that the chiral symmetry (2.4) of the Skyrme Lagrangian reduces to isospin symmetry, where $U_{R}=U_{L}^{-1}$. Equivalently, isospin symmetry is realized as an isospin rotation on the pion fields

$$
\pi_{i} \rightarrow \Omega_{i j} \pi_{j}
$$

Let us denote by $\mathscr{C}$ the configuration space of Skyrme fields at a given time which satisfy the boundary condition (2.7). The symmetry group acting on $\mathscr{C}$ is $\mathbb{E}_{3}$, the Euclidean group of $\mathbb{R}^{3}$, together with the group of isospin rotations, $S O(3)_{\text {isospin }}$. The operator quadratic form

$$
1-\frac{1}{4}\left[L_{i},\left[L_{i},\right]\right]
$$

derived from the kinetic energy expression (2.6), defines a Riemannian metric on the configuration space $\mathscr{C}$.

The Skyrme field equation is

$$
\partial_{\mu}\left(L^{\mu}+\frac{1}{4}\left[L_{\nu},\left[L^{\nu}, L^{\mu}\right]\right]\right)=0
$$

and the equation defining the gradient flow of a Skyrme field is

$$
L_{0}-\frac{1}{4}\left[L_{i},\left[L_{i}, L_{0}\right]\right]=\partial_{i}\left(L_{i}-\frac{1}{4}\left[L_{j},\left[L_{j}, L_{i}\right]\right]\right) .
$$

(The equation for the gradient flow on a finite-dimensional manifold with metric $g_{\alpha \beta}$ and potential $V$ is $g_{\alpha \beta}(\mathbf{q}) \dot{q}^{\beta}=-\partial_{\alpha} V$. Equation (2.11) is the analogue of this for the configuration space $\mathscr{C}$ ).

At a given time, $U\left(x_{0}, \mathbf{x}\right)$ is a map $\mathbb{R}^{3} \rightarrow S^{3}$, but because of (2.7), $\mathbb{R}^{3}$ may be compactified to a 3 -sphere of infinite radius. Then the map has a definite degree, an integer $B$, which Skyrme identified with the baryon number of the field. A formula for $B$ is

$$
B=\frac{1}{24 \pi^{2}} \int d^{3} x \varepsilon_{i j k} \operatorname{Tr}\left(L_{i} L_{j} L_{k}\right) .
$$


By continuity, $B$ is conserved, and this led Skyrme to the remarkable idea that physical baryons (proton, neutron etc.) may be modelled by solitons in a theory whose only fundamental fields represent pions.

A simple class of Skyrme fields are the spherically symmetric, time-independent hedgehog fields

$$
U(\mathbf{x})=\exp i f(r) \widehat{\mathbf{x}} \cdot \boldsymbol{\tau},
$$

where $r=|\mathbf{x}|$ and $\widehat{\mathbf{x}}=\mathbf{x} / r$. To satisfy the boundary condition (2.7) and ensure smoothness at the origin, one requires $f(r) \rightarrow 0$ as $r \rightarrow \infty$, and $f(0)=n \pi$ with $n$ an integer. (A shift of $f$ by an integer multiple of $2 \pi$ has no effect on $U$.) Such a hedgehog field has baryon number $B=n$.

For fields of hedgehog form the energy simplifies to

$$
V=4 \pi \int_{0}^{\infty} d r\left\{r^{2}\left(\frac{d f}{d r}\right)^{2}+2 \sin ^{2} f+\frac{1}{r^{2}} \sin ^{4} f+2 \sin ^{2} f\left(\frac{d f}{d r}\right)^{2}\right\}
$$

from which the variational equation for $f$ may be straightforwardly derived. Solutions can only be obtained numerically $[8,10]$. There appears to be a unique solution for each value of $n$, which minimizes the energy among fields of hedgehog form. The solutions for negative $n$ are obtained from those with positive $n$ by reversing the sign of $f$, and the energy is unchanged. For $n=0$ the solution is trivial. The solution for $n=1$ is known as the Skyrmion, and its energy is

$$
E_{1}=1.2314 \times 12 \pi^{2} .
$$

The solution for $n=2$ (the $B=2$ hedgehog solution) has energy

$$
E_{2}^{\text {hedgehog }}=1.834 \times 24 \pi^{2},
$$

which is significantly greater than $2 E_{1}$, and solutions for larger $n$ have considerably larger energy than $n E_{1}$. Hedgehog fields may be translated and rotated without changing their energy by replacing $\mathbf{x}$ by $\Omega(\mathbf{x}-\mathbf{X})$, where $\mathbf{X}$ is the new centre and $\Omega$ is a rotation matrix. Note that a rotation is equivalent to an isospin rotation. Numerical evidence strongly suggests that the Skyrmion is the minimal energy field of any shape with $B=1$. However, the $B=2$ hedgehog solution has been shown to be unstable to non-spherical deformations. The instabilities are discussed in detail in Sect. 6.

The asymptotic form of any hedgehog solution is

$$
f(r) \sim \frac{C}{4 \pi r^{2}}
$$

for some constant $C$. The asymptotic pion fields are therefore those of three mutually orthogonal scalar dipoles

$$
\pi_{i} \sim \frac{C x_{i}}{4 \pi r^{3}}
$$

each of dipole moment $C$. For the Skyrmion, $C \simeq 2.15$.

Let the Skyrmion centred at $\mathbf{X}$ and rotated by $\Omega$ be denoted $U(\mathbf{X}, \Omega)$. As Skyrme showed, one obtains a useful class of $B=2$ Skyrme fields by taking the product of two Skyrmions

$$
U=U\left(\mathbf{X}_{1}, \Omega_{1}\right) U\left(\mathbf{X}_{2}, \Omega_{2}\right)
$$


Provided $\left|\mathbf{X}_{2}-\mathbf{X}_{1}\right| \gg 1$, this product field models two well-separated, undistorted Skyrmions centred at $\mathbf{X}_{1}$ and $\mathbf{X}_{2}$, since each term in the product approaches 1 far from its centre. The energy of two well-separated Skyrmions differs from $2 E_{1}$ by the interaction energy of the pion dipoles. To leading order in the interaction, the total energy is [14]

$$
V=2 E_{1}-\frac{C^{2}}{4 \pi|\mathbf{X}|^{3}}\left(\Omega_{2} \Omega_{1}^{-1}\right)_{i j}\left(\delta_{\imath j}-3 \widehat{\mathbf{X}}_{i} \widehat{\mathbf{X}}_{j}\right),
$$

where $\mathbf{X}=\mathbf{X}_{2}-\mathbf{X}_{1}$. If $\Omega_{2} \Omega_{1}^{-1}$ is the matrix for a rotation by $\theta$ about an axis $\widehat{\mathbf{n}}$, then (2.20) may be rewritten as

$$
V=2 E_{1}-\frac{C^{2}}{4 \pi|\mathbf{X}|^{3}}(1-\cos \theta)\left(1-3(\widehat{\mathbf{X}} \cdot \widehat{\mathbf{n}})^{2}\right) .
$$

For fixed $\mathbf{X}$, the minimum energy occurs when $\theta=\pi$ and $\widehat{\mathbf{n}}$ is perpendicular to $\mathbf{X}$, that is, when one Skyrmion is rotated relative to the other by $\pi$ about an axis perpendicular to the line joining them. This defines the attractive channel for two Skyrmions. The maximum energy occurs when $\theta=\pi$ and $\hat{\mathbf{n}}$ is parallel to $\mathbf{X}$. There is a further saddle point of the energy, still with $\mathbf{X}$ fixed, when $\theta=0$ and the Skyrmions have the same orientation.

The manifold of such product fields is 12-dimensional, and has been used as a model for the two-Skyrmion moduli space [15]. However, a serious problem is that the minimal energy $B=2$ Skyrme fields, the $B=2$ toroids, are not even approximately of the product form. These latter fields, which are solutions of the Skyrme equation, were discovered by combining symmetry arguments with careful numerical work [9]. The solutions have axial symmetry, with energy concentrated in a toroidal region, and total energy

$$
E_{2}^{\text {toroid }} \simeq 1.18 \times 24 \pi^{2}
$$

which is about $4 \%$ less than $2 E_{1}$. Generally, translations, rotations and isospin rotations act independently on the $B=2$ toroids, but a rotation about the axis of symmetry has the same effect as an isospin rotation by twice the angle about a suitable "isoaxis". There is therefore an 8-dimensional family of $B=2$ toroids.

Summarizing: in the $B=1$ sector of the Skyrme model just one class of solutions is known-the Skyrmions. In the $B=2$ sector there are two classes of genuine solutions-the unstable high energy $B=2$ hedgehogs, and the minimal energy $B=2$ toroids. The two-Skyrmion moduli space $M_{12}$ will include these solutions and fields that interpolate between them. $M_{12}$ may be compactified by adding the virtual solutions, with intermediate energy, consisting of two infinitely separated Skyrmions.

Further remarkable solutions of the Skyrme field equation with $B=3,4,5$ and 6 have been found [16], and a spatially periodic solution representing a Skyrme crystal is also known [17], but we shall not discuss them here.

\section{Skyrme Fields from Instantons}

The basic formula which generates a Skyrme field in $\mathbb{R}^{3}$ from an instanton on $\mathbb{R}^{4}$ is [13]

$$
U(\mathbf{x})=\mathscr{P} \exp \int_{-\infty}^{\infty}-A_{4}(\mathbf{x}, t) d t .
$$


$A_{4}$ is the time-component of an $S U(2)$ Yang-Mills instanton [18], and $t\left(=x_{4}\right)$ is the auxillary Euclidean time, independent of the physical time $x_{0}$. The integral is along a line at fixed $\mathbf{x}$, parallel to the time axis, and $\mathscr{P}$ denotes path ordering. That is, $U(\mathbf{x})$ is the holonomy of the instanton along time-lines.

As is well known, instantons on $\mathbb{R}^{4}$ can be smoothly extended to $S^{4}$ by adding a point at infinity, but the resulting gauge potential may have an apparent singularity at infinity which can be removed by a gauge transformation. If $A_{4}$ tends to zero faster than $\left(|\mathbf{x}|^{2}+t^{2}\right)^{-1 / 2}$ then there is no singularity at infinity, and (3.1) as it stands defines a Skyrme field satisfying the boundary condition (2.7). $U(\mathbf{x})$ is the holonomy effectively along a closed curve, since all points at infinity in $\mathbb{R}^{4}$ are identified to one point on $S^{4}$. Provided we allow only gauge transformations that extend to $S^{4}$, the effect of a gauge transformation $g(\mathbf{x}, t)$ on $U(\mathbf{x})$ is simply a conjugation, or isospin rotation,

$$
U(\mathbf{x}) \rightarrow g_{\infty} U(\mathbf{x}) g_{\infty}^{-1}
$$

where $g_{\infty}$ is the asymptotic value of $g(\mathbf{x}, t)$.

If the instanton is in a singular gauge at infinity, a correction factor must be included in the definition (3.1) in order to obtain the true holonomy along closed curves in $S^{4}$. The correction, which is simply -1 in the case we shall study below, ensures that the Skyrme field $U(\mathbf{x})$ satisfies the boundary condition (2.7).

The fundamental topological result is that instantons of charge $k$ generate Skyrme fields of baryon number $k$. This follows from general principles. Recall that $S^{4}$ may be covered by two coordinate patches overlapping on an equatorial strip, and that an $S U(2)$ principle bundle over $S^{4}$ is characterized by a bundle transition function, a map $g$ from the equator $S^{3}$ to the group $S U(2)\left(=S^{3}\right)$. The topological charge of an instanton or any other gauge field on such a bundle is the degree of this map $k$. Now the space of gauge potentials on a given bundle is an affine space, and therefore connected. It follows by continuity that the baryon number of the Skyrme field generated from an instanton of charge $k$ equals the baryon number of the Skyrme field generated from any other gauge field on the same bundle. Now it is straightforward to construct a gauge potential whose component along meridians is zero. Consider the holonomy $U(\mathbf{x})$ of such a gauge potential along the closed meridional paths shown in Fig. 2, starting and ending at $N$, with $\mathbf{x}_{0}$ fixed and $\mathbf{x}$ variable. The gauge potential itself makes no contribution, so $U(\mathbf{x})$ equals $g^{-1}\left(\mathbf{x}_{0}\right) g(\mathbf{x})$. The map $U(\mathbf{x}): S^{3} \rightarrow S U(2)$ therefore has degree $k$. The result applies in particular to an instanton of charge $k$, and is gauge independent.

Now, the time-lines in $\mathbb{R}^{4}$, when mapped onto $S^{4}$, are conformally equivalent to meridians, with $N$ and $S$ tending to a single point. A similar result therefore holds: the Skyrme field $U(\mathbf{x})$ has baryon number $k$. The result may be checked by taking an example of an instanton of charge $k$, for which the Skyrme field $U(\mathbf{x})$ is simple to calculate. We shall exhibit such checks in what follows.

The $S U(2)$ instantons of charge $k$, with all gauge degrees of freedom quotiented out, have a moduli (parameter) space $I_{8 k-3}$ which is a connected manifold of dimension $8 k-3$ [19]. When we include the choice of gauge at infinity, the instantons have an $8 k$-dimensional moduli space. The resulting Skyrme fields depend on $8 k-1$ parameters, as a rigid time translation of the instanton leaves the Skyrme field unchanged. We denote the manifold of Skyrme fields generated by all instantons $\widetilde{M}_{8 k-1}$. It is a submanifold of the configuration space $\mathscr{C}$. Like $\mathscr{C}$, it is acted on by $\mathbb{E}_{3}$, the Euclidean group of $\mathbb{R}^{3}$, together with the isospin group $S O(3)_{\text {isospin }}$ which acts by conjugation on the Skyrme field, as in (3.2). 


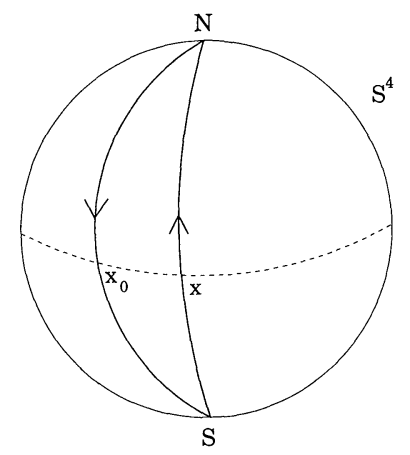

Fig. 2. Closed meridional paths on $S^{4}$. Along these paths, the holonomy of a gauge field of topological charge $k$ is a map of degree $k$

Some charge $k$ instantons can be regarded as $k$ well-separated single instantons. If these are well-separated in time, the Skyrme field that is generated is a product of $B=1$ Skyrme fields, ordered according to the time-ordering of the instantons. Even if some of the single instantons have small time separations, but large spatial separations, the resulting Skyrme field is still approximately of product form, because the ordering ambiguity is unimportant for Skyrme fields with little spatial overlap.

We have not been able to find an explicit formula for the Skyrme field $U(\mathbf{x})$ generated by a general instanton. It is an open problem to understand the properties of such a Skyrme field. Does it satisfy a differential equation, for example? We shall discuss in this paper only Skyrme fields generated by 't Hooft instantons [20,21], and the more general Jackiw, Nohl, Rebbi (JNR) instantons [21].

The time-component of a 't Hooft instanton of charge $k$ has the form

$$
A_{4}=\frac{i}{2} \frac{\nabla \varrho}{\varrho} \cdot \tau
$$

where

$$
\varrho(x)=1+\sum_{l=1}^{k} \frac{\lambda_{l}}{\left|x-X_{l}\right|^{2}} .
$$

The centres $\left\{X_{l}\right\}$ are arbitrary distinct points in $\mathbb{R}^{4}$, and the weights $\left\{\lambda_{l}\right\}$ are arbitrary positive constants. $A_{4}$ falls off sufficiently fast asymptotically, that (3.1) defines a Skyrme field. However, the gauge has been fixed in (3.3) and (3.4), so to obtain all Skyrme fields of interest, we need to include all conjugates $U_{0} U(\mathbf{x}) U_{0}^{-1}$ of the field $U(\mathbf{x})$ given by (3.1).

The integral (3.1) is apparently ill-defined for time-lines that pass through the singularities of $\varrho$, but the Skyrme field may instead be defined by taking the limit along any set of time-lines approaching the singular one. The result is unique and gives a smooth Skyrme field, because the apparent singularity in the instanton is only a gauge artifact.

A ' $t$ Hooft instanton of charge $k$ has $5 k$ parameters, and there are three more in $U_{0}$, but an overall time-translation does not affect the Skyrme field. Thus, these instantons generate a $(5 k+2)$-dimensional manifold of Skyrme fields. For $k=1$, but not $k>1$, every instanton can be gauge transformed to the 't Hooft form. 
A 't Hooft instanton with potential

$$
\varrho(\mathbf{x}, t)=1+\sum_{l=1}^{k} \frac{\lambda_{l}}{r^{2}+\left(t-T_{l}\right)^{2}}, \quad r=|\mathbf{x}|,
$$

(i.e. centres on the time axis), generates a baryon number $k$ Skyrme field of the hedgehog type (2.13). $A_{4}$ simplifies to the form

$$
A_{4}=i h(r, t) \widehat{\mathbf{x}} \cdot \tau
$$

so the path ordered integral (3.1) becomes an ordinary integral. The profile function $f(r)$ depends on the parameters $\left\{T_{l}, \lambda_{l}\right\}$ in a non-trivial way, via the roots of a polynomial (in $t$ ) of degree $2 k$. These roots can be explicitly found when $k=1$, and in the case of a time-reversal symmetric instanton when $k=2$ (see Sect. 5).

When $k=1$, since a time-translation has no effect, we can suppose

$$
\varrho(\mathbf{x}, t)=1+\frac{\lambda}{r^{2}+t^{2}} .
$$

Straightforward integration then gives a hedgehog field with profile function [13]

$$
f(r)=\pi\left[1-\left(1+\frac{\lambda}{r^{2}}\right)^{-1 / 2}\right] .
$$

As anticipated, $f(0)=\pi$ and $f(\infty)=0$, so $B=1$. The Skyrme energy function (2.14), for the profile (3.8), has a minimum value of

$$
\widetilde{E}_{1}=1.2432 \times 12 \pi^{2}
$$

when $\lambda=2.109$. For this value of $\lambda$, the field is similar to the Skyrmion solution of the Skyrme field equation, so in the context of instanton-generated Skyrme fields, we refer to this field as a Skyrmion. Notice that $\widetilde{E}_{1}$ exceeds $E_{1}$, given by $(2.15)$, by less than $1 \%$.

A charge $k$ 't Hooft instanton reduces to $k$ single instantons, centered at $\left\{X_{l}\right\}$ with scale sizes $\left\{\sqrt{\lambda_{l}}\right\}$, if the scale sizes are much less than the instanton separations. The resulting Skyrme field is then a product of $k$ separated $B=1$ hedgehogs, each with a profile of the form (3.8). The centre of the $l^{\text {th }}$ hedgehog is the spatial part of $X_{l}$ and its scale parameter is $\lambda=\lambda_{l}$. The major limitation of the 't Hooft instantons for our purpose is that all these hedgehogs have the same orientation.

The time-component of a JNR instanton of charge $k$ is again given by (3.3) but now

$$
\varrho(x)=\sum_{l=1}^{k+1} \frac{\lambda_{l}}{\left|x-X_{l}\right|^{2}}, \quad \lambda_{l}>0 .
$$

$A_{4}$ falls off only inversely with distance now, and the formula (3.1) can be shown to have limiting behaviour $U(\mathbf{x}) \rightarrow-1$ as $|\mathbf{x}| \rightarrow \infty$. It is therefore necessary to multiply (3.1) by -1 to obtain a Skyrme field. This factor may be interpreted as the extra holonomy along a large semicircle at infinity connecting $t=\infty$ with $t=-\infty$, which must be included to obtain holonomies along closed paths. It equals -1 , because the gauge potential of the JNR instanton is asymptotically $A_{\mu} \sim \partial_{\mu} g g^{-1}$, where $\lim _{t \rightarrow \infty} g=-\lim _{t \rightarrow-\infty} g$. The function $g$ may be interpreted as a bundle transition function defined on a 3 -sphere surrounding the point at infinity on $S^{4}$. 
In general, JNR instantons of charge $k$ depend on $5 k+4$ parameters, since a constant rescaling of the weights $\left\{\lambda_{l}\right\}$ has no effect. The resulting Skyrme fields depend on $5 k+6$ parameters, when allowance for time-translation and $U_{0}$ is made. However, when $k=1$ or $k=2$ there are fewer parameters. The case $k=2$ is the most interesting. Here, as observed first by Jackiw, Nohl and Rebbi [21], and elaborated by Hartshorne [22], one of the parameters is a gauge artifact, so the instantons have 13 parameters, and the resulting Skyrme fields have 15 parameters. This is the full complement of parameters for $k=2$ instantons, and indeed all $k=2$ instantons can be expressed in JNR form. In Sect. 4 we discuss the $k=2$ instantons in more detail.

The $k=1 \mathrm{JNR}$ instantons generate a 7-parameter family of hedgehog fields, the parameters determining the position, orientation and scale. This is because a JNR instanton with potential

$$
\varrho=\frac{\lambda_{1}}{\left|x-X_{1}\right|^{2}}+\frac{\lambda_{2}}{\left|x-X_{2}\right|^{2}}
$$

is gauge equivalent to a 't Hooft instanton with potential

$$
\varrho=1+\frac{\lambda}{|x-X|^{2}},
$$

where

$$
X=\frac{\lambda_{1} X_{2}+\lambda_{2} X_{1}}{\lambda_{1}+\lambda_{2}}
$$

and

$$
\lambda=\frac{\lambda_{1} \lambda_{2}}{\left(\lambda_{1}+\lambda_{2}\right)^{2}}\left|X_{2}-X_{1}\right|^{2} .
$$

The Skyrme fields generated by the JNR and 't Hooft forms of the $k=1$ instanton differ therefore only in their orientation. Explicitly, let $\tau=(\tau, 1)$ and let $\widehat{X}=\left(X_{2}-X_{1}\right) /\left|X_{2}-X_{1}\right|$. Then, as may be verified by calculating the asymptotic field, the Skyrme field generated by the JNR instanton is obtained from that of the 't Hooft instanton by conjugating by the $S U(2)$ matrix $U_{0}=\widehat{X} . \tau$.

\section{4. $k=2$ Instantons}

Hartshorne has shown that $k=2$ instantons have an elegant, geometrical, gauge invariant characterization [22]. It is simplest to describe this first for instantons on a 4-sphere, $S^{4}$. We regard $S^{4}$ as the unit sphere in $\mathbb{R}^{5}$ in the usual way. Hartshorne showed that there is a $1-1$ correspondence between $k=2$ instantons on $S^{4}$ (with all gauge freedom quotiented out) and a class of ellipses interior to $S^{4}$. Such an ellipse lies in a plane in $\mathbb{R}^{5}$ which intersects $S^{4}$ in a real circle. The admissible ellipses are those for which the ellipse and its associated circle satisfy the Poncelet condition, which means that there is a one-parameter family (or porism) of triangles with vertices on the circle and tangent to the ellipse. Poncelet proved, in fact, that if one such triangle exists, then there is automatically a porism of triangles and any point on the circle may be a vertex [23]. For a given circle, the Poncelet condition is a single algebraic constraint on the parameters of the ellipse.

For a given circle and ellipse there is an algebraic way of characterizing the porism of triangles inscribing the circle and tangent to the ellipse [22]. Introduce a projective 
linear coordinate $s$ along the circle. (In terms of a polar coordinate $\theta$, set $s=\tan \frac{1}{2} \theta$.) Associated with any triangle of the porism are the coordinates $s_{1}, s_{2}$ and $s_{3}$ of the vertices, and hence the cubic equation

$$
p_{0} s^{3}+p_{1} s^{2}+p_{2} s+p_{3}=0
$$

whose roots are $s_{1}, s_{2}$ and $s_{3}$. The coefficients $\left(p_{0}, p_{1}, p_{2}, p_{3}\right)$ are real, not all zero, and defined up to a constant factor, so they define a point in $\mathbb{R} P_{3}$. The fundamental property of the one-parameter family of cubics associated to the Poncelet porism is that their coefficients lie on a (projective) line in $\mathbb{R} P_{3}$. That is, if (4.1) is one of the cubics and

$$
q_{0} s^{3}+q_{1} s^{2}+q_{2} s+q_{3}=0
$$

with roots $s_{1}^{\prime}, s_{2}^{\prime}$ and $s_{3}^{\prime}$ is another, then the general cubic of the family is

$$
\left(\mu p_{0}+\nu q_{0}\right) s^{3}+\left(\mu p_{1}+\nu q_{1}\right) s^{2}+\left(\mu p_{2}+\nu q_{2}\right) s+\left(\mu p_{3}+\nu q_{3}\right)=0
$$

with $(\mu, \nu)$ real and not both zero. Note that the leading coefficient $\mu p_{0}+\nu q_{0}$ will be zero for some $(\mu, \nu)$, in which case the roots of (4.3) are taken to be $s=\infty$ together with the two roots of the surviving quadratic equation. $s=\infty$ corresponds to a finite point on the circle, so there is no problem. Note also that the geometrical requirement that the ellipse be interior to the circle corresponds to the algebraic statement that the roots of (4.3) are real and distinct for all $(\mu, \nu)$.

An immediate consequence of this algebraic approach is that if we know two triangles of the porism, possibly separated only infinitesimally, then we can determine the others by linear interpolation. The gauge invariant properties of the instanton can only depend on invariants of the line of cubics. We may, in fact, regard the instanton as defined by the circle and a line of cubics with real roots, since the resulting porism of triangles envelopes a unique ellipse.

Consider now the stereographic projection of $S^{4}$ (and its interior) from a point $N$ on the sphere to the $\mathbb{R}^{4}$ tangent to the sphere at the point $S$ antipodal to $N$. Since a stereographic projection is conformal, it maps a $k=2$ instanton on $S^{4}$ to a $k=2$ instanton on $\mathbb{R}^{4}$. There are two cases to consider, depending on whether the circle associated with the instanton on $S^{4}$ passes through $N$ or not. If it does not, then the circle, ellipse and associated porism of triangles in $\mathbb{R}^{5}$ project to a circle, ellipse and associated porism of triangles in a plane in $\mathbb{R}^{4}$. (The Poncelet condition is projectively invariant.) From the geometrical data in $\mathbb{R}^{4}$ one may reconstruct the instanton fields in JNR form. Choose one triangle of the porism (see Fig. 3) with vertices $X_{1}, X_{2}$ and $X_{3}$, and tangent to the ellipse at $A_{1}, A_{2}$ and $A_{3}$. Then define weights $\lambda_{1}, \lambda_{2}$ and $\lambda_{3}$, up to a common multiple, by

$$
\frac{\lambda_{1}}{\lambda_{2}}=\frac{X_{1} A_{3}}{A_{3} X_{2}}, \text { etc. }
$$

The JNR potential in $\mathbb{R}^{4}$ is given by

$$
\varrho=\frac{\lambda_{1}}{\left|x-X_{1}\right|^{2}}+\frac{\lambda_{2}}{\left|x-X_{2}\right|^{2}}+\frac{\lambda_{3}}{\left|x-X_{3}\right|^{2}},
$$

and this determines the gauge potential. In particular, the time-component of the gauge potential is, as before,

$$
A_{4}=\frac{i}{2} \frac{\nabla \varrho}{\varrho} \cdot \tau
$$




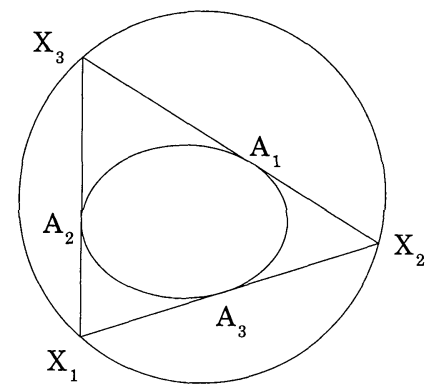

Fig. 3. The circle and ellipse associated with a $k=2$ instanton in $\mathbb{R}^{4}$, and one member of the porism of triangles with vertices on the circle and tangent to the ellipse

By pulling back the stereographic projection one obtains instanton fields on $S^{4}$. If another triangle of the porism is chosen, then a different expression for $\varrho$ is obtained, but the instanton changes only by a gauge transformation, and the Skyrme field it generates changes at most by an isospin rotation. The infinitesimal version of this gauge invariance was already noted by Jackiw, Nohl and Rebbi.

It is straightforward, conversely, to find the circle and ellipse in $\mathbb{R}^{4}$ associated with a $k=2$ instanton, given the JNR data. One defines $A_{1}, A_{2}$ and $A_{3}$ using (4.4) and then the circle is the circumcircle of the triangle $X_{1} X_{2} X_{3}$ and the ellipse is the conic tangent to the triangle at $A_{1}, A_{2}$ and $A_{3}$ (whose existence and uniqueness follow from the classical theory of conics [23]). An advantage of the geometrical data, because of its gauge invariance, is that it shows the symmetry of the instanton. The choice of a triangle implicit in the JNR potential can hide this. The Skyrme field generated from the instanton tends to have less symmetry than the instanton itself, because integration along time-lines breaks rotational symmetry in $\mathbb{R}^{4}$. The exception is where the plane containing the circle and ellipse either contains the time axis or is perpendicular to it.

The weights, as defined in (4.4), appear to be associated with one triangle of the porism, but they may be interpreted in terms of an infinitesimal motion of the triangle within the porism. For suppose $X_{1}^{\prime} X_{2}^{\prime} X_{3}^{\prime}$ is a triangle of the porism infinitesimally close to $X_{1} X_{2} X_{3}$. The side $X_{1} X_{2}$ rotates into $X_{1}^{\prime} X_{2}^{\prime}$ about the point of tangency to the ellipse $A_{3}$, and similarly for the other sides. Then, by elementary geometry, the ratio of lengths $X_{1} X_{1}^{\prime} / X_{2} X_{2}^{\prime}$ is related to the weights by

$$
\frac{X_{1} X_{1}^{\prime}}{X_{2} X_{2}^{\prime}}=\frac{X_{1} A_{3}}{A_{3} X_{2}}=\frac{\lambda_{1}}{\lambda_{2}} .
$$

Therefore, under an infinitesimal motion within the porism, the vertices $X_{i}$ move along the circle by a distance (or polar angle) proportional to $\lambda_{i}$. From this, one may see how the JNR data determine the line of cubics associated with a $k=2$ instanton. $X_{i} X_{i}^{\prime}$ is proportional to the change in polar angle $\delta \theta_{i}$ between $X_{i}$ and $X_{i}^{\prime}$. Now $s_{i}=\tan \frac{1}{2} \theta_{i}$, so $\delta s_{i}=\frac{1}{2}\left(1+s_{i}^{2}\right) \delta \theta_{i}$. Thus if one cubic of the porism has roots $s_{1}$, $s_{2}$ and $s_{3}$, then neighbouring ones have roots $s_{1}+\varepsilon \lambda_{1}\left(1+s_{1}^{2}\right), s_{2}+\varepsilon \lambda_{2}\left(1+s_{2}^{2}\right)$ and $s_{3}+\varepsilon \lambda_{3}\left(1+s_{3}^{2}\right)$, where $\varepsilon$ is infinitesimal. The line of cubics is therefore

$$
\begin{aligned}
& \mu\left(s-s_{1}\right)\left(s-s_{2}\right)\left(s-s_{3}\right) \\
& \quad+\nu\left(\lambda_{1}\left(1+s_{1}^{2}\right)\left(s-s_{2}\right)\left(s-s_{3}\right)+\lambda_{2}\left(1+s_{2}^{2}\right)\left(s-s_{3}\right)\left(s-s_{1}\right)\right. \\
& \left.\quad+\lambda_{3}\left(1+s_{3}^{2}\right)\left(s-s_{1}\right)\left(s-s_{2}\right)\right)=0 .
\end{aligned}
$$


This latter interpretation for the weights allows us to discuss the second case referred to above, where the circle on $S^{4}$ passes through $N$. Under stereographic projection, the instanton on $S^{4}$ still projects to an instanton on $\mathbb{R}^{4}$. This time the circle projects to a line $L$ in $\mathbb{R}^{4}$, and the ellipse, being coplanar with the circle, projects on to the same line and becomes degenerate. Because of the degeneracy it is no longer possible to keep track of tangents to the ellipse after projection. However, it is straightforward to consider the projection of the porism of triangles. The vertices of each triangle of the porism project to a triple of points on the line $L$. These triples are, as before, the roots of a line of cubics of the form (4.3), where $s$ is simply interpreted as an affine coordinate along $L$. Suppose one triple of points is $X_{1}, X_{2}$ and $X_{3}$, and suppose another triple, infinitesimally separated, is $X_{1}^{\prime}, X_{2}^{\prime}$ and $X_{3}^{\prime}$. Define weights, up to a multiple, by

$$
\frac{\lambda_{1}}{\lambda_{2}}=\frac{X_{1} X_{1}^{\prime}}{X_{2} X_{2}^{\prime}}, \text { etc. }
$$

Then the JNR potential is (4.5) as before, and the instanton fields may be calculated. Conversely, given a $k=2$ instanton in $\mathbb{R}^{4}$ with a JNR potential where $X_{1}, X_{2}$ and $X_{3}$ are collinear, one may invert the stereographic projection and reconstruct an instanton on $S^{4}$ and its associated circle and ellipse. An example is discussed in Sect. 8. In the collinear case, the JNR data determine the line of cubics in the following way. Suppose the points $X_{1}, X_{2}$ and $X_{3}$ on $L$ have affine coordinates $s_{1}, s_{2}$ and $s_{3}$. An infinitesimal motion along the porism changes these coordinates to $s_{1}+\varepsilon \lambda_{1}, s_{2}+\varepsilon \lambda_{2}$ and $s_{3}+\varepsilon \lambda_{3}$. The line of cubics is therefore

$$
\begin{aligned}
& \mu\left(s-s_{1}\right)\left(s-s_{2}\right)\left(s-s_{3}\right)+\nu\left(\lambda_{1}\left(s-s_{2}\right)\left(s-s_{3}\right)\right. \\
& \left.\quad+\lambda_{2}\left(s-s_{3}\right)\left(s-s_{1}\right)+\lambda_{3}\left(s-s_{1}\right)\left(s-s_{2}\right)\right)=0 .
\end{aligned}
$$

Let us conclude by mentioning a limiting case of $k=2$ instantons. Suppose, for an instanton on $S^{4}$, the associated Hartshorne ellipse has high eccentricity and almost touches $S^{4}$ at two points. The instanton degenerates to a pair of $k=1$ instantons near these points. After stereographic projection one obtains two wellseparated single instantons in $\mathbb{R}^{4}$. All possible separations and relative orientations occur. Let us describe this in more detail, using the JNR parametrization for instantons in $\mathbb{R}^{4}$, and assuming that $X_{1}, X_{2}$ and $X_{3}$ are not collinear. The relevant instantons are those where $\lambda_{1}=1$ (say) and $\lambda_{2} \ll 1, \lambda_{3} \ll 1$. Such data define an ellipse in $\mathbb{R}^{4}$ of large eccentricity, almost touching the circle through $X_{1}, X_{2}$ and $X_{3}$ at the points $X_{2}$ and $X_{3}$. In the neighbourhood of $X_{2}$ the JNR potential is approximately

$$
\varrho \simeq \frac{1}{\left|x-X_{1}\right|^{2}}+\frac{\lambda_{2}}{\left|x-X_{2}\right|^{2}}
$$

which gives a single instanton centred near $X_{2}$ (actually at $A_{3}$ ) with approximate scale parameter $\lambda_{2}\left|X_{2}-X_{1}\right|^{2}$ and orientation given by the direction of the line $X_{2} X_{1}$. Similarly there is a single instanton centred near $X_{3}$ with scale parameter $\lambda_{3}\left|X_{3}-X_{1}\right|^{2}$ and orientation given by the direction of $X_{3} X_{1}$. Since $\lambda_{2} \ll 1$ and $\lambda_{3} \ll 1$ the single instantons are small, relative to their separation. If the scales are appropriately fixed, then integration along the time-lines gives approximately a product of two Skyrmions, one located at the spatial position of $X_{2}$, the other at the spatial position of $X_{3}$, with orientations determined by those of the instantons.

In this limiting case, many triangles of the porism have $X_{2}$ and $X_{3}$ approximately fixed, and $X_{1}$ moving along the arc between $X_{2}$ and $X_{3}$. The single instantons centred near $X_{2}$ and $X_{3}$ retain their scales but change their orientations. However, because 
the angle $X_{2} X_{1} X_{3}$ remains approximately constant, the relative orientation of the instantons is unchanged, so the Skyrme field that is generated changes only by an isospin rotation, as expected. To see that two-Skyrmion product fields with all relative orientations can occur, consider $X_{1}$ in a position much nearer to $X_{2}$ than $X_{3}$, so that $\lambda_{3} \ll \lambda_{2} \ll 1$. Now keep $X_{2}, X_{3}, \lambda_{1}, \lambda_{2}$ and $\lambda_{3}$ all fixed, and allow $X_{1}$ to move freely on a 3-sphere of fixed radius centred at $X_{2}$. (The circle and ellipse will vary.) All orientations of the Skyrmion near the spatial location of $X_{2}$ occur (in fact twice over, as $S^{3}$ double covers the $S O(3)$ of orientations), whereas the orientation of the Skyrmion near the spatial location of $X_{3}$ remains approximately constant. All relative orientations therefore occur, twice each.

\section{Hedgehog Skyrme Fields from $k=2$ Instantons}

From $k=2$ instantons in $\mathbb{R}^{4}$ with $S O(3)$ rotational symmetry about the time axis we obtain a three-parameter family of $B=2$ Skyrme fields of the hedgehog type

$$
U(x)=\exp i f(r) \widehat{\mathbf{x}} \cdot \boldsymbol{\tau} .
$$

We have determined numerically the parameter values which give the minimal energy Skyrme field in this class; the energy exceeds by less than $2 \%$ the energy of the known solution of the Skyrme equation. In the first part of this section we show how to determine the profile function $f(r)$ from instanton data, and in the second part we present our results concerning the minimal energy field.

The hedgehog Skyrme field (5.1) is generated by a JNR instanton with the points $X_{1}, X_{2}$ and $X_{3}$ located on the time axis. The potential is of the form

$$
\varrho=\frac{\lambda_{1}}{r^{2}+\left(t-t_{1}\right)^{2}}+\frac{\lambda_{2}}{r^{2}+\left(t-t_{2}\right)^{2}}+\frac{\lambda_{3}}{r^{2}+\left(t-t_{3}\right)^{2}}, \quad \lambda_{i}>0
$$

which may be rewritten as

$$
\varrho=\frac{N(r, t)}{\left(r^{2}+\left(t-t_{1}\right)^{2}\right)\left(r^{2}+\left(t-t_{2}\right)^{2}\right)\left(r^{2}+\left(t-t_{3}\right)^{2}\right)},
$$

where $N$ is a positive quartic polynomial in $r$ and $t$. Apart from a constant factor, the quartic $N$ is gauge invariant, unlike the parameters $\left\{t_{i}, \lambda_{i}\right\}$, because it is an invariant of the line of cubics associated with the instanton. This is shown below. Let us regard $N$ as a quartic in $t$, with $r$-dependent coefficients, and denote its roots (which are gauge invariant and occur in complex conjugate pairs)

$$
\alpha(r), \quad \alpha^{*}(r), \quad \beta(r), \quad \beta^{*}(r)
$$

where, without loss of generality, $\operatorname{Im} \alpha>0$ and $\operatorname{Im} \beta>0$. Then

$$
\begin{aligned}
\log \varrho= & \log (t-\alpha)+\log \left(t-\alpha^{*}\right)+\log (t-\beta)+\log \left(t-\beta^{*}\right) \\
& -\sum_{j=1}^{3}\left[\log \left(t-t_{j}-i r\right)+\log \left(t-t_{j}+i r\right)\right],
\end{aligned}
$$

so

$$
\begin{aligned}
\frac{1}{\varrho} \frac{\partial \varrho}{\partial r}= & -\frac{1}{t-\alpha} \frac{d \alpha}{d r}-\frac{1}{t-\alpha^{*}} \frac{d \alpha^{*}}{d r}-\frac{1}{t-\beta} \frac{d \beta}{d r}-\frac{1}{t-\beta^{*}} \frac{d \beta^{*}}{d r} \\
& +i \sum_{j=1}^{3}\left[\frac{1}{t-t_{\jmath}-i r}-\frac{1}{t-t_{\jmath}+i r}\right] .
\end{aligned}
$$


Now, for an instanton with potential (5.3), the time-component of the gauge potential is

$$
A_{4}=\frac{i}{2}\left(\frac{1}{\varrho} \frac{\partial \varrho}{\partial r}\right) \widehat{\mathbf{x}} \cdot \tau .
$$

Along a time-line, $\widehat{\mathbf{x}} \cdot \boldsymbol{\tau}$ is a fixed element of the Lie algebra of $S U(2)$, so the path ordered integral (3.1) reduces to an ordinary integral. The resulting Skyrme field is of the hedgehog form (5.1), with profile function

$$
f(r)=-\frac{1}{2} \int_{-\infty}^{\infty} \frac{1}{\varrho} \frac{\partial \varrho}{\partial r} d t-\pi .
$$

$f(r)$ may be computed by contour integration. First note that because the leading terms in the polynomial $N$ are

$$
N \sim\left(\lambda_{1}+\lambda_{2}+\lambda_{3}\right) t^{4}-2\left(\lambda_{1}\left(t_{2}+t_{3}\right)+\lambda_{2}\left(t_{3}+t_{1}\right)+\lambda_{3}\left(t_{1}+t_{2}\right)\right) t^{3}+O\left(t^{2}\right),
$$

the sum of the roots is independent of $r$. Therefore, from (5.5), the integrand in (5.7) is $O\left(\frac{1}{t^{2}}\right)$ for large $|t|$, so the integral converges. After closing the contour of integration in the upper half $t$-plane, we find that

$$
f(r)=\pi i \frac{d}{d r}(\alpha+\beta)+2 \pi
$$

Again, because $\alpha+\alpha^{*}+\beta+\beta^{*}$ is independent of $r$, we obtain the final real expression

$$
f(r)=-\pi \frac{d}{d r}(\operatorname{Im} \alpha+\operatorname{Im} \beta)+2 \pi .
$$

Since the roots of a quartic can in principle be expressed in closed form, there is the possibility of finding a closed expression for $f(r)$, but the algebra is complicated and we have not pursued this route. Note that the numerator of $\varrho$ contributes the interesting part of $f(r)$. The denominator, which depends explicitly on $t_{1}, t_{2}$ and $t_{3}$ and is not gauge invariant, only contributes the unimportant constant $2 \pi$.

We verify that the profile function (5.10) is indeed that of a $B=2$ Skyrme field, by showing that $f(0)-f(\infty)=2 \pi$. For large $r$, the quartic $N(r, t)$ is

$$
N=\left(\lambda_{1}+\lambda_{2}+\lambda_{3}\right)\left(r^{2}+t^{2}\right)^{2}+\cdots
$$

and its roots are

$$
t=i r, i r,-i r,-i r
$$

with $O(1)$ corrections. Therefore $\alpha(r)=\beta(r)=i r$, and

$$
\lim _{r \rightarrow \infty} f(r)=0 \text {. }
$$

For small $r$, the roots of $N$ have the form const $+O\left(r^{2}\right)$. Therefore, at $r=0$,

$$
\frac{d}{d r}(\operatorname{Im} \alpha+\operatorname{Im} \beta)=0
$$

so $f(0)=2 \pi$. $f$ varies linearly with $r$ for small $r$, and as const $/ r^{2}$ for large $r$. 
Let us next clarify the relation between $N(r, t)$ and the line of cubics associated with the instanton. As we argued in Sect. 4, the line of cubics is

$$
\begin{aligned}
& \mu\left(t-t_{1}\right)\left(t-t_{2}\right)\left(t-t_{3}\right) \\
& \quad+\nu\left(\lambda_{1}\left(t-t_{2}\right)\left(t-t_{3}\right)+\lambda_{2}\left(t-t_{3}\right)\left(t-t_{1}\right)+\lambda_{3}\left(t-t_{1}\right)\left(t-t_{2}\right)\right)=0,
\end{aligned}
$$

where $(\mu, \nu)$ are homogeneous coordinates of the line. A general line of cubics $\mu p(t)+\nu q(t)=0$, where $p(t)=p_{0} t^{3}+p_{1} t^{2}+p_{2} t+p_{3}$ and $q(t)=q_{0} t^{3}+q_{1} t^{2}+q_{2} t+q_{3}$, has homogeneous Plücker coordinates

$$
l_{\mu \nu}=p_{\mu} q_{\nu}-p_{\nu} q_{\mu}
$$

It is straightforward to compute the Plücker coordinates $l_{\mu \nu}$ of the line (5.15). Further computation then shows that the quartic polynomial $N(r, t)$ has the following representation:

$$
\begin{aligned}
N(r, t)=\left\{l_{32} t^{4}+2 l_{31} t^{3}\right. & \left.+\left(l_{21}+3 l_{30}\right) t^{2}+2 l_{20} t+l_{10}\right\} \\
+\left\{2 l_{32} t^{2}\right. & \left.+2 l_{31} t+l_{21}-l_{30}\right\} r^{2} \\
& +l_{32} r^{4}
\end{aligned}
$$

This expression depends homogeneously on the Plücker coordinates, confirming that the roots of $N(r, t)$, and hence the associated profile function $f(r)$, depend only on the line of cubics and not on the particular cubic which one chooses in order to get an explicit potential of the form (5.2).

It is possibly useful to mention that the three polynomials in $t$ which occur as coefficients of $1, r^{2}$ and $r^{4}$ in (5.17) have a fairly simple interpretation as Jacobian invariants of the line of cubics (5.15). Indeed, for any cubics $p(t)$ and $q(t)$, one has the identities

$$
\begin{gathered}
l_{32} t^{4}+2 l_{31} t^{3}+\left(l_{21}+3 l_{30}\right) t^{2}+2 l_{20} t+l_{10}=\left|\begin{array}{cc}
q & p \\
\dot{q} & \dot{p}
\end{array}\right| \\
2 l_{32} t^{2}+2 l_{31} t+l_{21}-l_{30}=\frac{1}{2}\left|\begin{array}{cc}
\dot{q} & \dot{p} \\
\ddot{q} & \ddot{p}
\end{array}\right|-\frac{1}{6}\left|\begin{array}{cc}
q & p \\
\dddot{q} & \dddot{p}
\end{array}\right| \\
l_{32}=\frac{1}{12}\left|\begin{array}{cc}
\ddot{q} & \ddot{p} \\
\dddot{q} & \dddot{p}
\end{array}\right|
\end{gathered}
$$

where $\dot{p}=\frac{d p}{d t}$ etc. It would be nice if these observations led to a natural geometric interpretation for the Skyrme profile function $f(r)$.

An alternative to the JNR description of $k=2$ instantons with $S O(3)$ symmetry is 't Hooft's description. A 't Hooft instanton has $A_{4}$ given by (5.6), but the potential is now

$$
\varrho=1+\frac{\Lambda_{1}}{r^{2}+\left(t-T_{1}\right)^{2}}+\frac{\Lambda_{2}}{r^{2}+\left(t-T_{2}\right)^{2}}, \quad \Lambda_{i}>0
$$

which may be rewritten as

$$
\varrho=\frac{\tilde{N}(r, t)}{\left(r^{2}+\left(t-T_{1}\right)^{2}\right)\left(r^{2}+\left(t-T_{2}\right)^{2}\right)} .
$$

The presence of only two factors in the denominator of the 't Hooft potential means that the resulting gauge potential has better asymptotic behaviour than in the JNR case, so the appropriate definition of the Skyrme field is (3.1) without the extra factor of 
-1 . This is compensated by one fewer contribution of $\pi$ from the denominator. The result is that the hedgehog Skyrme field generated by a 't Hooft instanton with $S O(3)$ symmetry has a profile function of the form (5.10), with $\alpha$ and $\beta$ the roots of the quartic polynomial $\widetilde{N}(r, t)$.

A JNR instanton with potential (5.3) can be gauge transformed to the 't Hooft form by choosing that cubic on the line of cubics with one root, say $t_{3}$, at infinity, and then $t_{1}=T_{1}, t_{2}=T_{2}$. The singular factor in the denominator is then dropped to give (5.22). The numerator $\widetilde{N}(r, t)$ expressed in terms of $\Lambda_{1}, \Lambda_{2}, T_{1}, T_{2}$ is identical, up to a constant multiple, to the numerator $N(r, t)$ in (5.3). It is not difficult, given the JNR potential, to find the gauge equivalent 't Hooft parameters $\Lambda_{1}, \Lambda_{2}, T_{1}$ and $T_{2}$. One needs to find the line of cubics, and then find the cubic on the line whose leading coefficient is zero. $T_{1}$ and $T_{2}$ are the roots of the remaining quadratic polynomial. Then $\Lambda_{1}$ and $\Lambda_{2}$ are determined by linear algebra.

Conversely, given the 't Hooft potential (5.21), one can find the associated line of cubics as follows. One cubic on the line is

$$
\left(t-T_{1}\right)\left(t-T_{2}\right)=0 .
$$

The 't Hooft potential may be identified with a JNR potential (for all finite $t$ ) by taking $\lambda_{3} \rightarrow \infty, t_{3} \rightarrow \infty$ with $\lambda_{3} / t_{3}^{2}=1$, and then $t_{1}=T_{1}, \lambda_{1}=\Lambda_{1}, t_{2}=T_{2}$, $\lambda_{2}=\Lambda_{2}$. Now recall the JNR rule for shifting the roots, $\delta t_{i}=\varepsilon \lambda_{i}$. This rule implies here that

$$
\delta t_{1}=\varepsilon \Lambda_{1}, \quad \delta t_{2}=\varepsilon \Lambda_{2}, \quad \delta\left(\frac{1}{t_{3}}\right)=-\varepsilon \frac{\lambda_{3}}{t_{3}^{2}}=-\varepsilon .
$$

A cubic close to (5.23), with roots

$$
t_{1}=T_{1}+\varepsilon \Lambda_{1}, \quad t_{2}=T_{2}+\varepsilon \Lambda_{2}, \quad t_{3}=-\frac{1}{\varepsilon}
$$

is

$$
\left(t+\frac{1}{\varepsilon}\right)\left(t-T_{1}-\varepsilon \Lambda_{1}\right)\left(t-T_{2}-\varepsilon \Lambda_{2}\right)=0 .
$$

Expanding in $\varepsilon$, this becomes

$$
\left(t-T_{1}\right)\left(t-T_{2}\right)+\varepsilon\left(t\left(t-T_{1}\right)\left(t-T_{2}\right)-\Lambda_{1}\left(t-T_{2}\right)-\Lambda_{2}\left(t-T_{1}\right)\right)+O\left(\varepsilon^{2}\right)=0 .
$$

The line of cubics is therefore

$$
\mu\left(t-T_{1}\right)\left(t-T_{2}\right)+\nu\left(t\left(t-T_{1}\right)\left(t-T_{2}\right)-\Lambda_{1}\left(t-T_{2}\right)-\Lambda_{2}\left(t-T_{1}\right)\right)=0 .
$$

Let us consider next on how many parameters the hedgehog Skyrme fields depend. In the JNR potential there are effectively five parameters, as only $t_{1}, t_{2}, t_{3}$ and the ratios between $\lambda_{1}, \lambda_{2}$ and $\lambda_{3}$ are significant. However, another parameter drops out, because $t_{3}$, say, can be varied arbitrarily by moving along the line of cubics. Finally, the Skyrme field is unchanged by a rigid translation of $t_{1}, t_{2}$ and $t_{3}$ (which simply shifts $(\operatorname{Re} \alpha+\operatorname{Re} \beta)$ by a constant). We therefore have a three-parameter family of Skyrme fields. In the 't Hooft framework, the four parameters $T_{1}, T_{2}, \Lambda_{1}, \Lambda_{2}$ characterize the instanton, but the Skyrme field depends only on $T_{2}-T_{1}, \Lambda_{1}$ and $\Lambda_{2}$. The Skyrme field is actually invariant under the $\mathbb{Z}_{2}$ action $\left(\Lambda_{1}, \Lambda_{2}\right) \rightarrow\left(\Lambda_{2}, \Lambda_{1}\right)$, which corresponds to a time-reversal of the instanton, but we do not quotient out by this, because the action is not free (since $\Lambda_{1}=\Lambda_{2}$ is possible). 
An instanton may be centred on the time axis in a natural way by requiring $\operatorname{Re} \alpha+\operatorname{Re} \beta=0$. This condition is gauge invariant and $r$-independent. In the JNR potential one requires

$$
\lambda_{1}\left(t_{2}+t_{3}\right)+\lambda_{2}\left(t_{3}+t_{1}\right)+\lambda_{3}\left(t_{1}+t_{2}\right)=0
$$

and in the 't Hooft potential

$$
T_{1}+T_{2}=0
$$

Centring leads to no obvious simplification, however.

For time-reversal symmetric instantons, the formalism above simplifies and we obtain a closed expression for the Skyrme field, depending on two parameters. Let us use the 't Hooft parametrization here. We centre by setting $T_{1}=-T, T_{2}=T$ and impose time-reversal symmetry by setting $\Lambda_{1}=\Lambda_{2}=\Lambda$. The quartic polynomial $\widetilde{N}(r, t)$ reduces to a quadratic in $r^{2}$ and $t^{2}$

$$
\tilde{N}(r, t)=\left(r^{2}+t^{2}\right)^{2}+2\left(\Lambda+T^{2}\right) r^{2}+2\left(\Lambda-T^{2}\right) t^{2}+T^{4}+2 \Lambda T^{2} .
$$

It is straightforward to see that $\operatorname{Im} \alpha=\operatorname{Im} \beta$, and

$$
2(\operatorname{Im} \alpha)^{2}=r^{2}+\Lambda-T^{2}+\left(\left(r^{2}+\Lambda+T^{2}\right)^{2}-\Lambda^{2}\right)^{1 / 2} .
$$

The profile function is

$$
f(r)=2 \pi-2 \pi \frac{d}{d r}(\operatorname{Im} \alpha)
$$

We discuss next the energy of instanton-generated $B=2$ Skyrme fields of the hedgehog form. We present our results in terms of the 't Hooft parametrization. Recall that the energy expression for a hedgehog field with profile function $f$ is (2.14), and that the solution of the variational equation for $f$, satisfying the boundary conditions $f(0)=2 \pi, f(\infty)=0$, has energy $E_{2}^{\text {hedgehog }}=1.834 \times 24 \pi^{2}$.

It is not difficult to compute the energy for profile functions of the form (5.33), generated by time-reversal symmetric instantons. Preliminary work, reported in [13], suggested that the minimum occurred in the limit $T \rightarrow \infty$, where the profile function has the simple form

$$
f(r)=2 \pi\left[1-\left(1+\frac{\Lambda}{r^{2}}\right)^{-1 / 2}\right] .
$$

Varying $\Lambda$, one finds a minimal energy $E=1.855536 \times 24 \pi^{2}$ when $\Lambda=2.6211$. More careful calculations give, however, a different result. We now find a minimal energy of $E=1.855529 \times 24 \pi^{2}$ when $T^{2}=84.6$ and $\Lambda=2.6427 .^{1}$ This slight reduction in the energy has been verified by treating the profile at large, finite $T^{2}$ as a perturbation of the profile (5.34).

It is satisfactory that the minimal energy obtained from the instanton-generated profile is less than $2 \%$ greater than the energy of the true solution. Practically, it makes hardly any difference from an energetic point of view whether $T^{2}=84.6$ or $T^{2}=\infty$. Conceptually, it is better that the minimum occurs for finite $T$ and $\Lambda$ for this means that the energy has a quadratic minimum in the interior of the parameter space, rather than a linear minimum at the edge.

\footnotetext{
1 We are grateful to Robert Leese for confirming the results of these calculations using a different algorithm, and for improving their numerical accuracy
} 
It is more difficult to analyse the instantons which are not time-reversal symmetric. As we have seen, $f(r)$ depends on the derivatives of the roots of the polynomial $\widetilde{N}(r, t)$, and we have not attempted to calculate these. Nor have we calculated $f(r)$ from the integral (5.7). Instead we have used an approximation. The general 't Hooft potential, after centring, may be written

$$
\varrho=1+\frac{\Lambda+\Delta}{r^{2}+(t-T)^{2}}+\frac{\Lambda-\Delta}{r^{2}+(t+T)^{2}}, \quad \Delta<\Lambda .
$$

If $T^{2} \gg \Lambda$, the resulting Skyrme field has approximate profile

$$
f(r)=2 \pi-\pi\left(1+\frac{\Lambda+\Delta}{r^{2}}\right)^{-\frac{1}{2}}-\pi\left(1+\frac{\Lambda-\Delta}{r^{2}}\right)^{-\frac{1}{2}},
$$

which is the profile of the product of two instanton-generated $B=1$ hedgehogs, with scale parameters $\Lambda+\Delta$ and $\Lambda-\Delta$. Essentially, (5.36) allows for time-reversal asymmetry but assumes $T=\infty$. We have evaluated the energy for this profile as a function of $\Lambda$ and $\Delta$. The minimum is at $\Delta=0$, and with $\Lambda=2.6211$, $E=1.855536 \times 24 \pi^{2}$ as before. The energy is symmetric in $\Delta$ (both for the potential (5.35) and for the approximate profile (5.36)), so it is reasonable to conjecture that when $T^{2}=84.6$, which is large compared with $\Lambda$ when $\Lambda=2.6427$, the minimal energy for the exact profile still occurs when $\Delta=0$. If so, then $E=1.855529 \times 24 \pi^{2}$ is the minimal energy of $B=2$ hedgehog Skyrme fields obtained from $k=2$ instantons. We shall assume from now on that this conjecture is true.

\section{Instabilities of $B=2$ Hedgehogs}

The spherically symmetric $B=2$ hedgehog solution of the Skyrme field equation (in the standard form (2.13)) is stable against perturbations which preserve the hedgehog form, but is not stable against other perturbations. Its instability is not surprising, as its energy is considerably greater than that of two well-separated Skyrmions. Wirzba and Bang have determined the unstable modes [11]. They span a six-dimensional vector space, which decomposes under the orthogonal group $O(3)$ into two irreducible threedimensional spaces. $O(3)$ acts by the vector representation on one of these, and by the axial vector representation on the other.

The product ansatz gives an interpretation of these unstable modes. The product of two $B=1$ Skyrmions, with both oriented in the standard way and both centred at the origin, gives a good approximation to the $B=2$ hedgehog solution. The energy is reduced if either the centers are separated, or the relative orientation is changed. Separation of the Skyrmions gives an axial vector perturbation of the $B=2$ hedgehog, the vector being along the separation direction. Rotating one Skyrmion relative to the other gives a vector perturbation, the vector being along the axis of rotation. These perturbations are qualitatively like the modes found by Wirzba and Bang. They have the same angular structure but somewhat different dependence on the radial coordinate. Naively, one might have expected that vector and axial vector would have occurred the other way round here, but they do not because the ordering in the product ansatz is important when one considers reflections.

If the instanton-generated $B=2$ Skyrme fields are to be useful, then the instantongenerated analogue of the $B=2$ hedgehog solution should have six similar unstable modes. Now we described in the last section the minimal energy $B=2$ hedgehog 


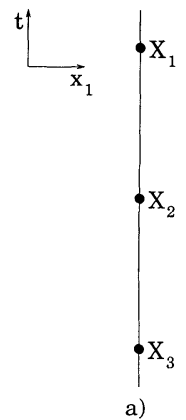

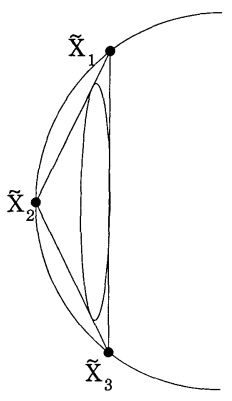

b)

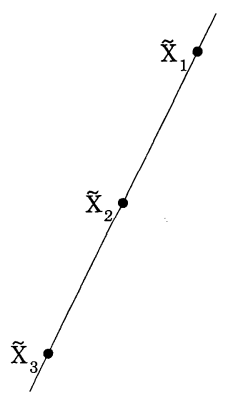

c)

Fig. 4a-c. Instanton data for a the minimal energy $B=2$ hedgehog field, $\mathbf{b}$ the same, perturbed by its vector unstable mode $\mathbf{c}$ the same, perturbed by its axial vector unstable mode

field generated from $k=2$ instantons. It has three energy-increasing modes preserving the hedgehog form. It also has six energy-neutral modes (zero modes) associated with translations and rotations (or isospin rotations). There remain six instanton-generated modes which are potentially energy-decreasing. We have not investigated their effect on the energy directly, as this is computationally hard. Instead we shall show here that they qualitatively perturb the field in the same way as Wirzba and Bang's negative modes, and we conjecture that they decrease the energy in the same way.

Recall that in the ' $t$ Hooft parametrization, the potential of the instanton generating the $B=2$ hedgehog of minimal energy is

$$
\varrho=1+\frac{\Lambda}{r^{2}+(t-T)^{2}}+\frac{\Lambda}{r^{2}+(t+T)^{2}},
$$

with $T^{2}=84.6, \Lambda=2.6427$. It is convenient here to transform to the JNR form (i.e. choose another cubic along the line of cubics associated with (6.1)), and bring one root of the cubic from $\infty$ to 0 . The resulting potential, which is still time-reversal symmetric, is

$$
\varrho=\frac{1}{r^{2}+t^{2}}+\frac{\Lambda^{\prime}}{r^{2}+\left(t-T^{\prime}\right)^{2}}+\frac{\Lambda^{\prime}}{r^{2}+\left(t+T^{\prime}\right)^{2}} .
$$

The relation of $\left(T^{\prime}, \Lambda^{\prime}\right)$ to $(T, \Lambda)$ is easily found by expressing (6.1) and (6.2) over common denominators, and comparing numerators. One finds $T^{\prime}=\left(T^{2}+2 \Lambda\right)^{1 / 2}$ and $\Lambda^{\prime}=\Lambda / T^{2}$. The JNR points $X_{1}, X_{2}$ and $X_{3}$ for the minimal energy $B=2$ hedgehog are shown in Fig. 4a.

One perturbation of the JNR data is indicated in Fig. 4b. $X_{1}$ and $X_{3}$ move by purely spatial displacements in the $x_{1}$-direction to $\widetilde{X}_{1}$ and $\widetilde{X}_{3}$, and $\tilde{X}_{2}$ coincides with $X_{2}$. The weights $\lambda_{1}, \lambda_{2}$ and $\lambda_{3}$ are unchanged to leading order. The vector character is measured by (for example) the spatial displacement of $\widetilde{X}_{3}$ from $X_{3}$. The perturbed Skyrme field is not symmetric under $x_{1} \rightarrow-x_{1}$, but it has $O(2)$ symmetry in the $x_{2}-x_{3}$ plane (the same symmetry as a vector along the $x_{1}$-axis). An axial vector perturbation is obtained by spatially perturbing $X_{1}$ and $X_{3}$ in the $x_{1}$-direction to the positions $\tilde{X}_{1}$ and $\widetilde{X}_{3}$ in Fig. 4c, leaving $X_{2}$ fixed. Again the spatial displacement of $\tilde{X}_{3}$ from $X_{3}$ is a measure of the perturbation. The perturbed Skyrme field has $S O(2)$ symmetry in the $x_{2}-x_{3}$ plane as well as the reflection symmetry under 
$x_{1} \rightarrow-x_{1}$. A reflection $x_{2} \rightarrow-x_{2}$ reverses the sign of the perturbation, because it arises from simultaneous reflections $t \rightarrow-t$ and $x_{2} \rightarrow-x_{2}$ of the instanton data. (These symmetry properties are the same as for an axial vector along the $x_{1}$-axis.) If these perturbations are combined, with both vectors in the $x_{1}$-direction, one obtains a rather general instanton with its circle in the $x_{1}-t$ plane. The only symmetry of the resulting Skyrme field is $S O(2)$. If the perturbations are combined, with the vector in the $x_{1}$-direction and axial vector in the $x_{2}$-direction, then the instanton has data as in Fig. $4 \mathrm{~b}$ but tilted towards the $x_{2}$-direction. The resulting Skyrme field has no continuous symmetry.

Our arguments so far show that the perturbations associated with varying the instanton moduli as in Fig. $4 \mathrm{~b}$ and $4 \mathrm{c}$ have the same symmetry properties as the energy-decreasing perturbations of the $B=2$ hedgehog solution of the Skyrme equation. This suggests that these perturbations decrease the energy among instantongenerated fields. But the argument can be made a bit more convincing. Note that the parameters of the potential (6.1) are such that there are two well-separated single instantons along the time axis, and the resulting Skyrme field is approximately a product of two coincident Skyrmions with the same orientation. Now, the instanton whose JNR points are as in Fig. $4 \mathrm{~b}$ also gives a $B=2$ Skyrme field approximately of product type. The Skyrmions have the same spatial location but they no longer have the same orientation (see the discussion of fields of product type in Sect. 4). Similarly, the instanton of Fig. 4c gives a product of two Skyrmions with the same orientation but different positions. It follows that the perturbations of the instanton moduli we are discussing have approximately the effect of changing the positions and orientations of two Skyrmions in a product ansatz, and are therefore likely to decrease the energy.

\section{Two Skyrmions - The Attractive Channel}

Two Skyrmions oriented to minimize their energy at a given separation are said to be in the attractive channel. The field configurations in this channel are quite well understood from several viewpoints. Verbaarschot et al. [24] argued that the fields in the attractive channel have no continuous symmetries but have three mutually orthogonal reflection symmetries, and a typical field has an energy density schematically as in Fig. 5, with reflection symmetries in the three coordinate planes. At large separation, the two Skyrmions are hardly distorted from their spherical form, but one is rotated relative to the other by $180^{\circ}$ about the $x_{3}$-axis (with the labelling of axes in Fig. 5). As they approach, they deform, and at minimal separation the Skyrmions coalesce into a $B=2$ toroid whose field has $O(2)$ symmetry in the $x_{1}-x_{2}$ plane as well as reflection symmetry under $x_{3} \rightarrow-x_{3}$.

Verbaarschot et al. defined a Skyrmion separation parameter (a moment of the baryon density) for $B=2$ Skyrme fields with the assumed symmetries. Then, using a Lagrange multiplier technique, they found numerically the minimal energy field at various separations. As the separation decreases, the energy decreases monotonically from $2 E_{1}$, the energy of two infinitely separated Skyrmions, to $E_{2}^{\text {toroid }}$. Walhout has recently repeated the calculation with a different definition of the separation parameter, with qualitatively similar results [25]. Any definition of separation is rather ad hoc, and it would be interesting if the gradient flow curve connecting infinitely separated Skyrmions to the toroid could be found, but it is unlikely to be very different from the paths through the constrained minima found in $[24,25]$. 


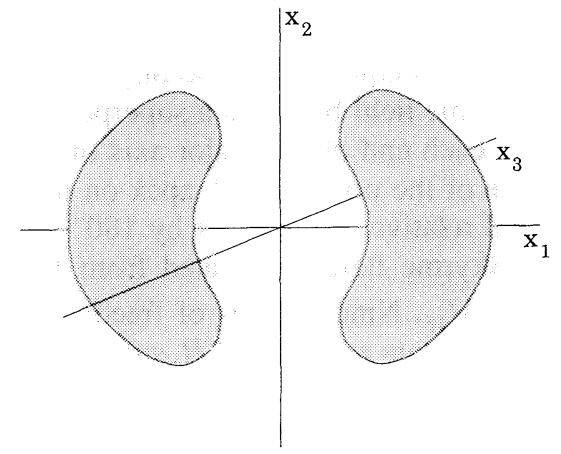

Fig. 5. Regions where the energy of two separated Skyrmions in the attractive channel is concentrated (shown schematically)

Let us assume that such a gradient flow curve exists. Then the moduli space of $B=2$ Skyrme fields $M_{12}$, introduced in Sect. 1, has a 10-dimensional subspace of attractive channel fields, which we denote $M_{10}$. The generic orbits of the symmetry group $\mathbb{E}_{3} \times S O(3)_{\text {isospin }}$ acting on $M_{10}$ are 9-dimensional, and the tenth coordinate is a measure of the separation. The orbit of the minimal energy toroids is exceptional. Because of the $O(2)$ symmetry of a toroid, the orbit is 8-dimensional.

Skyrme fields generated by instantons model the attractive channel well, at all separations. The geometrical data in $\mathbb{R}^{4}$ of the relevant instantons are a concentric circle and ellipse in a spatial plane. Suppose the major and minor axes of the ellipse are $a$ and $b$, and the radius of the circle is $R$. It is straightforward to verify that there is a porism of triangles with vertices on the circle and tangent to the ellipse if and only if $a+b=R$. If the circle and ellipse are located as in Fig. 6, then the energy density is as in Fig. 5. There are manifestly three reflection symmetries in the $x_{1^{-}}$, $x_{2^{-}}$, and $x_{3}$-axes. These axes are distinguished from each other, but they have no preferred orientation.

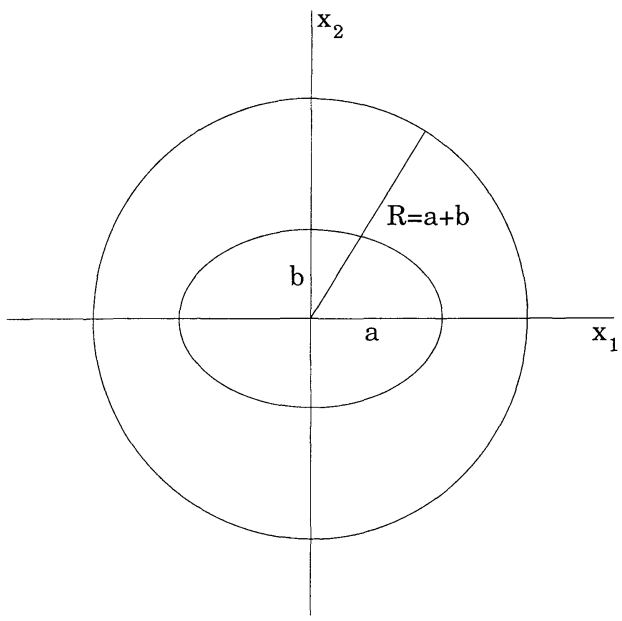

Fig. 6. Circle and ellipse associated with a $k=2$ instanton which generates an attractive channel Skyrme field. 
A special case is when $a=b=\frac{1}{2} R$. Then the instanton has circular symmetry and the triangles of the porism are all equilateral. The Skyrme field that is generated is a toroid. When $a \gg b$, the Skyrme field becomes a superposition of two well-separated $B=1$ hedgehogs, one near each end of the major axis of the ellipse. Using the JNR data for an isosceles triangle of the porism, with apex on the $x_{2}$-axis, one may verify that one hedgehog is rotated relative to the other by $180^{\circ}$ about the $x_{3}$-axis.

The attractive channel Skyrme fields generated from $k=2$ instantons form an 11-dimensional subspace of $\widetilde{M}_{15}$. Nine degrees of freedom arise from the symmetry group, and the remaining parameters are $a, b$ and $R$, subject to the constraint $a+b=R$. Ideally, one should find the gradient flow curve in the $(a, R)$ parameter space which descends from infinitely separated Skyrmions, i.e. hedgehogs of energy minimizing size, to a minimal energy toroid (with $a=\frac{1}{2} R$ ). Its orbit under the symmetry group would be a space $\widetilde{M}_{10}$, which would model $M_{10}$. The gradient flow curve has not been found, but a qualitatively similar path of constrained minima has been, by Hosaka et al. [26]. They have calculated numerically the energy of Skyrme fields obtained from these instantons (and others). They used the JNR formula for the instanton, associated with one of the isosceles triangles of the porism. Here two of the reflection symmetries are manifest, and the third was verified algebraically. For a given ratio $a / b$ (or eccentricity of the ellipse) the Skyrme energy depends on the scale $R$ in a simple way. The energy is minimized when the sigma-model and Skyrme term parts of the energy are equal. Hosaka et al. showed that this minimal energy decreases as $a / b$ decreases, reaching an overall minimum when $a / b=1$. By introducing a (again, rather ad hoc) separation parameter, they inferred that the energy decreases as the separation decreases. At all separations the instanton-generated fields have $1 \%-2 \%$ greater energy than those found in [24, 25].

Let us now describe more geometrically the manifolds of attractive channel Skyrme fields $M_{10}$ and $\widetilde{M}_{10}$. To simplify the discussion, let us quotient out the free action of the group of translations and isospin rotations. There remain 4-dimensional manifolds of centred Skyrme fields, $M_{4}$ and $\widetilde{M}_{4}$, acted on by $S O(3)$, the group of spatial rotations. Either of these manifolds is parametrized by a separation coordinate, and by three Euler angles which specify a frame of labelled but unoriented Cartesian axes. The generic $S O(3)$ orbit is therefore $S O(3) / V$, where $V$ is the viergruppe of $180^{\circ}$ rotations about three Cartesian axes. At minimal separation, the orbit is $\mathbb{R} P_{2}$, the set of unoriented symmetry axes of minimal energy toroids. There is a smooth manifold with this orbit structure, namely the Atiyah-Hitchin manifold (AH) parametrizing centred two-monopole solutions of the $S U(2)$ Bogomol'nyi equations [4]. It is therefore not necessary to consider Skyrme fields where the separation parameter is less than its value at the toroids, as in [24, 25]. In the attractive channel, Skyrmions can approach head-on and scatter smoothly through $90^{\circ}$ (as has recently been verified in a numerical solution of the Skyrme equation [27]). The major and minor axes of the ellipse exchange roles in the instanton description, preserving the reflection symmetries.

It appears, therefore, that $\mathrm{AH}$ is the manifold parametrizing centred Skyrme fields in the attractive channel. The metric and potential energy must be calculated using the Skyrme Lagrangian restricted to the appropriate fields. If one regards the fields calculated in [24-26] as good approximations to those on $M_{4}$ and $\widetilde{M}_{4}$, then some of this dynamical information is known. The angular part of the metric has been determined by Verbaarschot et al. and by Walhout, as they calculated moments of 
inertia of their field configurations, but unfortunately the data is not presented in detail. The radial part of the metric has not been calculated. For a given set of fields, the precise definition of the separation parameter will no longer matter once the radial metric is determined. The potential energy is simply the static energy of the Skyrme field, as a function of separation.

A candidate metric on $M_{4}$ or $\widetilde{M}_{4}$ is the Atiyah-Hitchin metric of monopole theory [4]; it has the correct qualitative behaviour but cannot be correct in detail. Skyrmions have a weaker asymptotic interaction than the velocity-dependent Coulomb interaction between monopoles, implicit in the Atiyah-Hitchin metric.

The manifold AH may be compactified by including the $\mathbb{R} P_{2}$ representing Skyrmions with infinite separation. This is a topological compactification, and the metric must be modified. A point in $\mathbb{R} P_{2}$ specifies the (unoriented) line of separation of the Skyrmions, but the rotation angle about this line is lost. The resulting manifold is $S^{4}$. The foliation of $S^{4}$ by $S O(3)$ with generic orbit $S O(3) / V$ and two special $\mathbb{R} P_{2}$ orbits is familiar in various contexts.

The manifold $\mathrm{AH}$ is not simply connected, but it has a simply connected double cover $\widehat{\mathrm{AH}}$ which may also be compactified by adding $\mathbb{R} P_{2}$ at infinity. The resulting manifold is $\mathbb{C} P_{2}$. (For a discussion of $\mathbb{C} P_{2}$ as a branched double cover of $S^{4}$, and of the action of $S O(3)$ on these manifolds, see [28].) Now Skyrmions should be quantized as fermions, and it has been shown that two-Skyrmion wavefunctions, for Skyrmions restricted to the attractive channel, are well defined only on the covering space $\widehat{\mathrm{AH}}[12,24,25]$. For this reason, both $\mathbb{C} P_{2}$ and $S^{4}$ should be considered when discussing Skyrmions in the attractive channel.

We may summarize as follows. The moduli space of centred two-Skyrmion fields in the attractive channel, with isospin rotations quotiented out, appears to be a smooth 4-dimensional manifold similar to the Atiyah-Hitchin manifold, and with the same $S O(3)$ orbit structure. It would best be found using gradient flow curves, but this remains to be done. Various approximations to the manifold have been computed, using constrained minimization applied to general Skyrme fields and instantongenerated Skyrme fields. In these approximations the potential energy is known as a function of a separation parameter, but the Riemannian geometry is not yet known in detail.

\section{Ellipses, $\mathbb{C} P_{4}$ and the Global Picture}

We have argued that the space $\widetilde{M}_{15}$ of $B=2$ Skyrme fields generated by $k=2$ instantons accurately models various types of low and moderate energy Skyrme fields. From now on, we assume that the gradient flow of the Skyrme energy function, restricted to these fields, defines a subspace $\widetilde{M}_{12}$ which is qualitatively the same as, and quantitatively close to, the two-Skyrmion moduli space $M_{12}$. After quotienting out by the free action of the group $\mathbb{R}^{3} \times S O(3)_{\text {isospin }}$, there remains a 6-dimensional space $\widetilde{M}_{6}$. For the remainder of this paper we shall discuss only $\widetilde{M}_{6}$.

The picture we have developed of the stationary points of the Skyrme energy function on $\widetilde{M}_{6}$, and of the gradient flow, is as follows. The energy has a maximum at a point representing a minimal energy $B=2$ hedgehog. The tangent space to $\widetilde{M}_{6}$ here is the 6-dimensional space of unstable modes discussed in Sect. 6. There is an $\mathbb{R} P_{2}$ of minima, a point in $\mathbb{R} P_{2}$ specifying the unoriented axis of symmetry of a 
minimal energy $B=2$ toroid. Finally, if $\widetilde{M}_{6}$ is compactified, there is an $\mathbb{R} P_{2}$ of saddle points representing two infinitely separated Skyrmions, a point in this $\mathbb{R} P_{2}$ specifying the unoriented direction of separation. Each of these saddle points has two negative modes, which bring the Skyrmions to a finite separation in the attractive channel. Almost all gradient flow curves which descend from the maximum end at one of the minima, but some exceptional curves end at a saddle point. The 4-dimensional manifold AH representing attractive channel fields is a submanifold of $\widetilde{M}_{6}$, and is the union of gradient flow curves from the saddle points down to the minima.

This information about $\widetilde{M}_{6}$ does not immediately tell us its global structure. For a more detailed understanding, let us consider the global geometry of the moduli space $I_{13}$ of $k=2$ instantons, where all gauge freedom has been quotiented out. It is best to consider the instantons to be defined on $S^{4}$, the unit 4-sphere in $\mathbb{R}^{5}$ with centre $O$. Then an instanton is completely characterized by its Hartshorne ellipse, and there is a 1-1 correspondence between $I_{13}$ and the ellipses interior to $S^{4}$ which satisfy the Poncelet condition with their associated coplanar circles on $S^{4}$. There is a large symmetry group acting on the instantons and hence on the ellipses, namely $S O(5,1)$, the conformal group of $S^{4}$. Note that the interior of $S^{4}$ can be identified with hyperbolic 5-space, $H^{5}$, the lines in $H^{5}$ being the Euclidean line segments connecting two points on $S^{4}$. The group $S O(5,1)$ acts on $H^{5}$ as the isometry group of the hyperbolic metric. A Hartshorne ellipse may be interpreted as an ellipse in $H^{5}$, and has a well-defined hyperbolic centre [29]. The ellipse and its hyperbolic centre are carried round $H^{5}$ naturally by the action of $S O(5,1)$, and when the hyperbolic centre is at $O$, but not otherwise, the usual euclidean centre of the ellipse is also at $O$.

Now fix the point $N$ on $S^{4}$ to be $(0,0,0,0,1)$ in $\mathbb{R}^{5}$. Stereographic projection from $N$ is on to the $\mathbb{R}^{4}$ defined by $x_{5}=-1$. Consider the subgroup $\mathbb{R}^{4} \beth \mathbb{R}^{+}$of the conformal group $S O(5,1)$ which keeps $N$ fixed, and preserves the directions of tangent vectors at $N$. This subgroup becomes the semi-direct product of translations and rescalings of instantons after the instantons are stereographically projected from $N$. The orbit of a Hartshorne ellipse under this subgroup has precisely one representative whose centre is at $O$. This is because the group $\left.\mathbb{R}^{4}\right] \mathbb{R}^{+}$acts transitively and freely on $H^{5}$, so the hyperbolic centre of the Hartshorne ellipse is moved to $O$ by a unique element of the group. $I_{13}$ therefore has the structure

$$
I_{13}=C_{8} \times \mathbb{R}^{4} \times \mathbb{R}^{+}
$$

where $C_{8}$ is the 8-dimensional manifold of Hartshorne ellipses centred at $O$.

We recall now a standard argument, which shows that the manifold $C_{8}$ is $\left(\mathbb{C} P_{4}-\mathbb{R} P_{4}\right) / *$, where $/ *$ means modulo complex conjugation. Consider an oriented ellipse in $\mathbb{R}^{5}$ centred at the origin, with major axis a and minor axis $\mathbf{b}$. There is an associated element of $\mathbb{C} P_{4}$ with homogeneous coordinates $\mathbf{Z}=\mathbf{a}+i \mathbf{b}$. If the ellipse is not degenerate, then $\mathbf{Z}$ is not in $\mathbb{R} P_{4}$. Note that ellipses with different scales are identified to the same point in $\mathbb{C} P_{4}$. One may replace $(\mathbf{a}, \mathbf{b})$ by $(-\mathbf{a},-\mathbf{b})$ without changing the orientation, but this leads to the same element of $\mathbb{C} P_{4}$. If the ellipse is in fact a circle, then $|\mathbf{a}|=|\mathbf{b}|$ and $\mathbf{a} \cdot \mathbf{b}=0$, and one may replace $(\mathbf{a}, \mathbf{b})$ by $(\mathbf{a} \cos \alpha+\mathbf{b} \sin \alpha, \mathbf{b} \cos \alpha-\mathbf{a} \sin \alpha)$. Then $\mathbf{Z}$ is replaced by $\mathbf{Z}^{\prime}=(\mathbf{a}+i \mathbf{b}) e^{-i \alpha}=\mathbf{Z} e^{-i \alpha}$, which is again the same point in $\mathbb{C} P_{4}$. An oriented ellipse in $\mathbb{R}^{5}$, of undetermined scale, and centred at the origin, is therefore associated with a well-defined element of $\mathbb{C} P_{4}-\mathbb{R} P_{4}$. For an unoriented ellipse, $(\mathbf{a}, \mathbf{b})$ is identified with $(\mathbf{a},-\mathbf{b})$, so that unoriented ellipses are associated with elements of $\left(\mathbb{C} P_{4}-\mathbb{R} P_{4}\right) / *$. 
Conversely, suppose we are given an element of $\mathbb{C} P_{4}-\mathbb{R} P_{4}$, denoted in homogeneous coordinates by a complex 5 -vector $\mathbf{Z}=\mathbf{a}+i \mathbf{b}$. There are two cases to consider. If $\mathbf{Z}^{2}=|\mathbf{a}|^{2}-|\mathbf{b}|^{2}+2 i \mathbf{a} \cdot \mathbf{b}=0$ then identify $\mathbf{a}$ and $\mathbf{b}$ with orthogonal radii of a circle centred at the origin. The scale ambiguity of $\mathbf{Z}$ means that the circle has an arbitrary size; the phase ambiguity leads to an arbitrariness in the choice of $(\mathbf{a}, \mathbf{b})$ in the plane of the circle, but the circle itself and its orientation are unambiguous. If $\mathbf{Z}^{2} \neq 0$, multiply $\mathbf{Z}$ by a phase factor $e^{i \alpha}$ so that $\mathbf{Z}^{2}$ is real and positive. Then $\mathbf{a} \cdot \mathbf{b}=0$ and $|\mathbf{a}|>|\mathbf{b}|$. Since $\mathbf{Z}$ is not in $\mathbb{R} P_{4}$, both $\mathbf{a}$ and $\mathbf{b}$ are non-zero. $\mathbf{a}$ and $\mathbf{b}$ may therefore be identified with the major and minor axes of an ellipse. The remaining arbitrariness in $\mathbf{Z}$ is its scale and its sign (since $\alpha$ can be replaced by $\alpha+\pi$ ), which means that the scale of the ellipse is undetermined, and $(\mathbf{a}, \mathbf{b})$ is identified with $(-\mathbf{a},-\mathbf{b})$. So $\mathbb{C} P_{4}-\mathbb{R} P_{4}$ parametrizes centred, oriented ellipses of undetermined scale in $\mathbb{R}^{5}$. (More generally, $\mathbb{C} P_{n-1}-\mathbb{R} P_{n-1}$ parametrizes centred, oriented ellipses of undetermined scale in $\mathbb{R}^{n}$, for any $n \geq 2$.)

Now the Hartshorne ellipses are unoriented, and their scale is determined by the Poncelet condition. For ellipses centred at $O$, the Poncelet condition is $|\mathbf{a}|+|\mathbf{b}|=1$. Clearly, among a 1-parameter family of centred ellipses, which differ only in their scale, there is precisely one which satisfies this Poncelet condition. We conclude that $C_{8}$ may be identified with $\left(\mathbb{C} P_{4}-\mathbb{R} P_{4}\right) / *$.

Having found the form of $I_{13}$, we now quotient out the factor $\mathbb{R}^{4}$ which parametrizes translations. This is because the Skyrme fields generated by instantons are independent of a time-translation, and in $\widetilde{M}_{6}$ we have quotiented out the spatial translations. Also, since $I_{13}$ parametrizes instantons with no residual gauge freedom, the Skyrme fields they generate have their isospin rotations quotiented out, as in $\widetilde{M}_{6}$. Our task therefore reduces to identifying $\widetilde{M}_{6}$ as a subspace of $C_{8} \times \mathbb{R}^{+}$.

The instantons parametrized by a fixed element $c$ of $C_{8}$ and a variable scale parameter in $\mathbb{R}^{+}$generate Skyrme fields in $\mathbb{R}^{3}$ which differ only in scale. The terms of the Skyrme energy function vary in a simple way under rescaling - the sigma model term varies directly with the scale, and the Skyrme term inversely. There is therefore a unique scale $l(c)$ at which the sum of these terms is minimized. The pairs $(c, l(c))$ define a graph in $C_{8} \times \mathbb{R}^{+}$which is diffeomorphic to $C_{8}$. Let us identify the graph with $C_{8}$, which parametrizes Hartshorne ellipses centred at $O$, but we must remember now that there is a scale factor in $\mathbb{R}^{+}$associated with such an ellipse. The stationary points of the Skyrme energy function on $C_{8} \times \mathbb{R}^{+}$lie on the graph, and we expect the gradient flow between them to lie either on or close to the graph. We conjecture that if we restrict the Skyrme Lagrangian and the associated gradient flow to the graph, then we shall find a good approximation to $\widetilde{M}_{6}$, with the correct global structure. The results of Hosaka et al. [26] show that this conjecture appears to be verified for the attractive channel fields.

It is convenient at this point to consider the simply connected double cover of $\widetilde{M}_{6}$, by supposing that centred Hartshorne ellipses are oriented, and parametrized by elements of $\mathbb{C} P_{4}-\mathbb{R} P_{4}$. A given instanton is therefore associated with a pair of ellipses of opposite orientations. We shall comment on the physical relevance of orientability later. The elements of $\mathbb{C} P_{4}-\mathbb{R} P_{4}$ which parametrize the minimal energy $B=2$ hedgehog and attractive channel Skyrme fields are as follows.

The minimal energy hedgehog, which is a maximum on $\widetilde{M}_{6}$, was described in Sect. 5. The associated instanton in $\mathbb{R}^{4}$ defines a porism of triples of points along the $t$-axis. Two triples of the porism are $(-T, T, \infty)$ and $\left(-T^{\prime}, 0, T^{\prime}\right)$, where $T^{2}=84.6$, 
$T^{2}=T^{2}+2 \Lambda$, and $\Lambda=2.6427$. After inverse stereographic projection, these points are mapped to the unit circle on $S^{4}$ lying in the $x_{4}-x_{5}$ plane, and become the vertices of two triangles. Let us use $t$ as a projective linear coordinate on the circle, so the coordinates of the vertices of the triangles are the same as those of the triples on the line. The two triangles envelope a unique Hartshorne ellipse interior to the circle. Since $T \gg 1$, this ellipse is not centred at $O$. Let us now rescale by an element of $\mathbb{R}^{+}$to centre the ellipse. The effect is to multiply $T$ and $T^{\prime}$ by a common factor $\kappa$, so the pair of triangles have vertices at $(-\kappa T, \kappa T, \infty)$ and $\left(-\kappa T^{\prime}, 0, \kappa T^{\prime}\right)$. The ellipse is centred when these triangles are congruent, i.e. when $\kappa^{2} T T^{\prime}=1$. The major and minor axes of the centred ellipse are $\mathbf{a}=\left(0,0,0,1-b_{0}, 0\right)$ and $\mathbf{b}=\left(0,0,0,0, b_{0}\right)$, where the signs have been fixed arbitrarily. Because $T^{2} \gg \Lambda$, we find an ellipse of high eccentricity, with $b_{0} \simeq 0.015$. The elements of $\mathbb{C} P_{4}$ corresponding to the two orientations of this ellipse are $\mathbf{Z}=\left(0,0,0,1-b_{0}, \pm i b_{0}\right)$.

An attractive channel Skyrme field is generated by an instanton in $\mathbb{R}^{4}$ whose geometric data are a concentric circle and ellipse in a spatial plane. Since we have quotiented out by translations, we may assume the centres of the circle and ellipse are at the origin of $\mathbb{R}^{4}$. After inverse stereographic projection the circle and ellipse are still concentric, and in a plane orthogonal to the $x_{4}$ - and $x_{5}$-axes. By rescaling by an element of $\mathbb{R}^{+}$, we can centre this Hartshorne ellipse at $O$. The element of $\mathbb{C} P_{4}$ parametrizing the centred ellipse is of the form $\mathbf{Z}=\left(a_{1}+i b_{1}, a_{2}+i b_{2}, a_{3}+i b_{3}, 0,0\right)$, where $\mathbf{a}$ and $\mathbf{b}$ are, up to a scale factor, the major and minor axes of the original ellipse in $\mathbb{R}^{4}$. The attractive channel Skyrme fields are thus parametrized by the subspace $\mathbb{C} P_{2}-\mathbb{R} P_{2}$ of $\mathbb{C} P_{4}-\mathbb{R} P_{4}$, defined by $z_{4}=z_{5}=0$. This subspace is a concrete model for the manifold $\widetilde{\mathrm{AH}}$ discussed in Sect. 7. The minimal energy toroidal Skyrme fields are those where the Hartshorne ellipse is a circle, and are parametrized by the elements $\mathbf{Z}$ of $\mathbb{C} P_{2}$ for which $\mathbf{Z}^{2}=0$, that is, by the (complex) conic $Q_{1}$. As we argued before, the compactification of the space of attractive channel Skyrme fields, obtained by including infinitely separated Skyrme fields, is $\mathbb{C} P_{2}$.

Elements of $\mathbb{C} P_{4}$ of the form $\mathbf{Z}=\left(0,0,0, z_{4}, z_{5}\right)$ parametrize instantons whose JNR points $X_{1}, X_{2}$ and $X_{3}$ (after stereographic projection to $\mathbb{R}^{4}$ ) are on the $t$-axis. Variations of the real and imaginary parts of $z_{5} / z_{4}$ away from $z_{5} / z_{4}= \pm i b_{0} /\left(1-b_{0}\right)$, together with variations of the $\mathbb{R}^{+}$scale parameter, generate perturbations of the minimal energy $B=2$ hedgehog which preserve its hedgehog form and increase its energy. The unstable modes of the hedgehog are orthogonal to these and are therefore generated by instantons whose $\mathbb{C} P_{4}$ parameters are of the form

$$
\mathbf{Z}=\left(z_{1}, z_{2}, z_{3}, 1-b_{0}, \pm i b_{0}\right),
$$

where $\left|z_{1}\right|,\left|z_{2}\right|$ and $\left|z_{3}\right|$ are small. If $\left(z_{1}, z_{2}, z_{3}\right)$ is a real 3 -vector, the perturbation is of the type indicated by Fig. 4c. If $\left(z_{1}, z_{2}, z_{3}\right)$ is a pure imaginary 3-vector, the perturbation is of the type indicated by Fig. $4 \mathrm{~b}$.

We have now given the $\mathbb{C} P_{4}$ parameters for the minimal energy $B=2$ hedgehog field, and for the attractive channel fields, and (8.2) gives the parameters for the unstable directions away from the hedgehog. The gradient flow of the Skyrme energy function joins the hedgehog to the attractive channel fields. Since we have not computed the gradient flow curves, let us consider the simplest model for this join, which is given by linear interpolation. Let $\mathbb{C P}_{3}^{+}$denote the complex projective 3-space which linearly joins the point $\left(0,0,0,1-b_{0}, i b_{0}\right)$ to the projective plane $\left(\mathbb{C} P_{2}\right)$ defined by $z_{4}=z_{5}=0$. The interpolating points in $\mathbb{C} P_{3}^{+}$are of the form $\left(z_{1}, z_{2}, z_{3}, 1-b_{0}, i b_{0}\right)$, where $z_{1}, z_{2}$ and $z_{3}$ are arbitrary complex numbers. $\mathbb{C} P_{3}^{+}$has the desired tangent space 
at $\left(0,0,0,1-b_{0}, i b_{0}\right)$. Let $\mathbb{C} P_{3}^{-}$similarly denote the linear join of $\left(0,0,0,1-b_{0},-i b_{0}\right)$ to the same $\mathbb{C} P_{2}$. $\mathbb{C} P_{3}^{+}$and $\mathbb{C} P_{3}^{-}$intersect on the $\mathbb{C} P_{2}$, so their union $\mathbb{C} P_{3}^{+} \cup \mathbb{C} P_{3}^{-}$ is not smooth. The only points in $\mathbb{C} P_{3}^{+} \cup \mathbb{C} P_{3}^{-}$which represent infinitely separated Skyrmions are those in the $\mathbb{R} P_{2}$ subspace of the common $\mathbb{C} P_{2}$, where $z_{2} / z_{1}$ and $z_{3} / z_{1}$ are real. Therefore, the space $\left(\mathbb{C} P_{3}^{+} \cup \mathbb{C} P_{3}^{-}\right)-\mathbb{R} P_{2}$ represents two Skyrmions which are either coincident or at finite separation.

We now state our main conjecture. This is that the double cover of $\widetilde{M}_{6}$ is diffeomorphic to the space $\left(\mathbb{C} P_{3}^{+} \cup \mathbb{C} P_{3}^{-}\right)-\mathbb{R} P_{2}$. The only likely difference from the linear model is that $z_{4}$ and $z_{5}$ vary somewhat nonlinearly as $z_{1}, z_{2}$ and $z_{3}$ vary, but with the $S O(3)$ symmetry acting on $z_{1}, z_{2}$ and $z_{3}$ preserved. As stated above, the linear model has the correct tangent space at the points $\left(0,0,0,1-b_{0}, \pm i b_{0}\right)$. The gradient flow on the double cover of $\widetilde{M}_{6}$ is incomplete, as some gradient flow curves begin and some end with two Skyrmions infinitely separated. By including the $\mathbb{R} P_{2}$ representing infinitely separated Skyrmions, we obtain a space diffeomorphic to $\mathbb{C} P_{3}^{+} \cup \mathbb{C} P_{3}^{-}$. This is compact, and the gradient flow is complete.

The individual gradient flow curves on the double cover of $\widetilde{M}_{6}$ cannot be exactly or even approximately linear, because most of them end on the conic $Q_{1}$ of $\mathbb{C} P_{2}$, defined by $z_{1}^{2}+z_{2}^{2}+z_{3}^{2}=0$. However, the Skyrme energy varies very little on the $\mathbb{C} P_{2}$ of attractive channel fields, relative to the energy difference between the hedgehog and toroids. It is therefore possible that a typical gradient flow curve is approximately a line until it gets close to the $\mathbb{C} P_{2}$, and then it turns and ends at a point of $Q_{1}$.

A model energy function on $\mathbb{C} P_{4}$ which gives this type of gradient flow is

$$
\begin{aligned}
E= & \frac{\left|z_{4}\right|^{2} \times \phi\left(z_{5} / z_{4}\right)}{\left|z_{1}\right|^{2}+\left|z_{2}\right|^{2}+\left|z_{3}\right|^{2}+\left|z_{4}\right|^{2}+\left|z_{5}\right|^{2}} \\
& +\varepsilon \frac{\left|z_{1}^{2}+z_{2}^{2}+z_{3}^{2}\right|^{2}}{\left(\left|z_{1}\right|^{2}+\left|z_{2}\right|^{2}+\left|z_{3}\right|^{2}+\left|z_{4}\right|^{2}+\left|z_{5}\right|^{2}\right)^{2}}
\end{aligned}
$$

where $\varepsilon$ is small and positive, and $\phi$ has a minimal value of 1 where $z_{5} / z_{4}=$ $\pm i b_{0} /\left(1-b_{0}\right) . E$ has saddle points where $z_{1}=z_{2}=z_{3}=0$ and $z_{5} / z_{4}= \pm i b_{0} /\left(1-b_{0}\right)$, corresponding to the minimal energy $B=2$ hedgehog. There are lower saddle points where $z_{4}=z_{5}=0$ and $\left(z_{1}, z_{2}, z_{3}\right)$ is a real point of $\mathbb{C} P_{2}$ (i.e. $z_{2} / z_{1}$ and $z_{3} / z_{1}$ are real). These represent infinitely separated Skyrmions. Finally, there are minima where $z_{4}=z_{5}=0$ and $z_{1}^{2}+z_{2}^{2}+z_{3}^{2}=0$. These represent the minimal energy toroids. With the standard metric on $\mathbb{C} P_{4}$, the union of the $\mathbb{C} P_{3}$ 's defined by $z_{5} / z_{4}= \pm i b_{0} /\left(1-b_{0}\right)$ is invariant under the gradient flow, and linearly models the compactified double cover of $\widetilde{M}_{6}$. If $\varepsilon=0$, the gradient flow is along lines, but if $\varepsilon$ is small and positive, the gradient flow on either one of the $\mathbb{C} P_{3}$ 's is as in Fig. 1.

One may exploit the $S O(3)$ symmetry to pick out some invariant subspaces of the gradient flow. For example, the subspace $z_{3}=0$ is invariant, and almost all gradient flow curves in it descend to $(1, i, 0,0,0)$ or $(1,-i, 0,0,0)$. These points both represent a toroid with symmetry axis along the $x_{3}$-axis, but with opposite orientations. An example of a smaller invariant subspace is $z_{2}=z_{3}=0$. Here, gradient flow curves descend to $(1,0,0,0,0)$, which represents two Skyrmions infinitely separated along the $x_{1}$-axis. The lines within this subspace, with either $z_{1}$ real or $z_{1}$ pure imaginary, are also gradient flow curves. Finally, because of the reflection symmetry $z_{5} \rightarrow-z_{5}$, the $\mathbb{C} P_{2}$ submanifold $z_{4}=z_{5}=0$, which represents the attractive channel, is invariant. Within it, $S O(3)$ symmetry implies that all the gradient flow curves are lines of the 
form $\left(\lambda a_{1}+i b_{1}, \lambda a_{2}+i b_{2}, \lambda a_{3}+i b_{3}, 0,0\right)$ where $\mathbf{a}$ and $\mathbf{b}$ are orthogonal vectors and $\lambda$ varies from $\infty$ to 1 . These lines connect a pair of infinitely separated Skyrmions to a toroid.

The pair of (possibly nonlinear) subspaces of $\mathbb{C} P_{4}$ discussed above, $\mathbb{C} P_{3}^{+}$and $\mathbb{C} P_{3}^{-}$, are mapped into each other by complex conjugation. Since Hartshorne ellipses are really unoriented, we should identify conjugate points. In particular, we should identify the points $\left(z_{1}, z_{2}, z_{3}, 0,0\right)$ and $\left(z_{1}^{*}, z_{2}^{*}, z_{3}^{*}, 0,0\right)$ in the $\mathbb{C} P_{2}$ where $\mathbb{C} P_{3}^{+}$and $\mathbb{C} P_{3}^{-}$intersect. Our conjecture for the structure of the double cover of $\widetilde{M}_{6}$ therefore implies that $\widetilde{M}_{6}$ itself is diffeomorphic to $\left(\mathbb{C} P_{3} / *\right)-\mathbb{R} P_{2}$, and its compactification is diffeomorphic to $\mathbb{C} P_{3} / *$. The operation $*$ does not act in the standard way on $\mathbb{C} P_{3}$, but only acts on the $\mathbb{C} P_{2}$ subspace $z_{4}=z_{5}=0$. The fixed point set of the operation * is the $\mathbb{R} P_{2}$ representing infinitely separated Skyrmions. $\mathbb{C} P_{3} / *$ is not a smooth manifold but is rather like a manifold, embedded in a larger space, which intersects itself. There are two distinct tangent spaces at the points of intersection.

In the quantized theory of Skyrmions, it is necessary to consider wavefunctions on the double cover of the two-Skyrmion moduli space, which takes us back to oriented ellipses. Fermionic quantization of Skyrmions requires that in a quantum state where the total momentum and isospin are zero (i.e. where the wavefunction descends to the double cover of $\widetilde{M}_{6}$ ), the wavefunction changes sign under change of orientation, or equivalently $\mathbf{Z} \rightarrow \mathbf{Z}^{*}$. The allowed wavefunctions are therefore determined by their values on one copy (say $\mathbb{C} P_{3}^{+}$) of $\mathbb{C} P_{3}$, and they are unconstrained there, except that they must change sign under $*$ on the $\mathbb{C} P_{2}$ where $\mathbb{C} P_{3}^{+}$and $\mathbb{C} P_{3}^{-}$intersect.

The symmetry $z_{5} \rightarrow-z_{5}$ also maps $\mathbb{C} P_{3}^{+}$to $\mathbb{C} P_{3}^{-}$, and allows a finer classification of the allowed wavefunctions. Energy eigenstates may be assumed to be either even or odd under this symmetry. Wavefunctions that are even change sign under $\left(z_{1}, z_{2}, z_{3}\right) \rightarrow\left(z_{1}^{*}, z_{2}^{*}, z_{3}^{*}\right)$ over all of $\mathbb{C} P_{3}^{+}$, and may be non-zero on the $\mathbb{C} P_{2}$ representing attractive channel fields. Wavefunctions that are odd are unchanged under $\left(z_{1}, z_{2}, z_{3}\right) \rightarrow\left(z_{1}^{*}, z_{2}^{*}, z_{3}^{*}\right)$, and must therefore vanish on the $\mathbb{C} P_{2}$. Low energy states, like the deuteron, will have even wavefunctions. An even wavefunction must vanish at the point $\left(0,0,0,1-b_{0}, i b_{0}\right)$ representing the minimal energy $B=2$ hedgehog. For the deuteron state, such a result is not unexpected. The deuteron has isospin zero and spin 1, which also implies that the wavefunction vanishes at the hedgehog.

It remains a considerable task to calculate in detail the gradient flow of the Skyrme energy function on the space of instanton-generated $B=2$ Skyrme fields, and to check whether our picture of $\widetilde{M}_{6}$, and hence of the two-Skyrmion moduli space $\widetilde{M}_{12}$, is correct. It is also important to check that $\widetilde{M}_{12}$ is a good approximation to the space $M_{12}$. If all is well, then one may use $\widetilde{M}_{12}$ with confidence to investigate the classical and quantized dynamics of two Skyrmions.

\section{References}

1. Bogomol'nyi, E.B.: The stability of classical solutions. Sov. J. Nucl. Phys. 24, 449 (1976)

2. Weinberg, E.: Parameter counting for multimonopole solutions. Phys. Rev. D20, 936 (1979)

3. Taubes, C.H.: Arbitrary $N$-vortex solutions of the first order Ginzburg-Landau equations. Commun. Math. Phys. 72, 277 (1980)

4. Manton, N.S.: A remark on the scattering of BPS monopoles. Phys. Lett. 110B, 54 (1982) Atiyah, M.F., Hitchin, N.J.: The geometry and dynamics of magnetic monopoles, Princeton, NJ: Princeton University Press 1988 
Bates,L., Montgomery, R.: Closed geodesics on the space of stable two-monopoles. Commun. Math. Phys. 118, 635 (1988)

Temple-Raston, M.: Closed 2-dyon orbits. Nucl. Phys. B313, 447 (1989)

Leese, R.: Low energy scattering of solitons in the $\mathbb{C} P_{1}$ model. Nucl. Phys. B344, 33 (1990)

Samols, T.M.: Vortex scattering. Commun. Math. Phys. 145, 149 (1992)

5. Gibbons, G.W., Manton, N.S.: Classical and quantum dynamics of BPS monopoles. Nucl. Phys. B274, 183 (1986)

Schroers, B.J.: Quantum scattering of BPS monopoles at low energy. Nucl. Phys. B367, 177 (1991)

6. Manton, N.S.: Unstable manifolds and soliton dynamics. Phys. Rev. Lett. 60, 1916 (1988)

7. Skyrme, T.R.H.: A unified field theory of mesons and baryons. Nucl. Phys. 31, 556 (1962) Nyman, E.M., Riska, D.O.: Low energy properties of baryons in the Skyrme model. Reps. Prog. Phys. 53, 1137 (1990)

8. Adkins, G.S., Nappi, C.R., Witten, E.: Static properties of nucleons in the Skyrme model. Nucl. Phys. B228, 552 (1983)

9. Kopeliovich, V.B., Stern, B.E.: Exotic Skyrmions. JETP Lett. 45, 203 (1987)

Verbaarschot, J.J.M.: Axial symmetry of bound baryon number-two solution of the Skyrme model. Phys. Lett. 195B, 235 (1987)

Schramm, A.J., Dothan, Y., Biedenharn, L.C.: A calculation of the deuteron as a biskyrmion. Phys. Lett. 205B, 151 (1988)

10. Jackson, A.D., Rho, M.: Baryons as chiral solitons. Phys. Rev. Lett. 51, 751 (1983)

11. Wirzba, A., Bang, H.: The mode spectrum and the stability analysis of Skyrmions on a 3-sphere. Nucl. Phys. A515, 571 (1990)

Zenkin, S.V., Kopeliovich, V.B., Stern, B.E.: The soliton interaction in the Skyrme model. Sov. J. Nucl. Phys. 45, 106 (1987)

12. Braaten, E., Carson L.: Deuteron as a toroidal Skyrmion. Phys. Rev. D38, 3525 (1988)

13. Atiyah, M.F., Manton, N.S.: Skyrmions from instantons. Phys. Lett. 222B, 438 (1989)

Manton, N.S.: Skyrme fields and instantons. In: Geometry of low dimensional manifolds: 1 (LMS Lecture Notes 150), Donaldson, S.K., and Thomas C.B., eds. Cambridge: Cambridge University Press 1990

14. Jackson, A., Jackson, A.D., Pasquier, V.: The Skyrmion-Skyrmion interaction. Nucl. Phys. A432, 567 (1985)

15. Vinh Mau, R., Lacombe, M., Loiseau, B., Cottingham, W.N., Lisboa, P.: The static baryonbaryon potential in the Skyrme model. Phys. Lett. 150B, 259 (1985)

16. Braaten, E., Townsend, S., Carson, L.: Novel structure of static multisoliton solutions in the Skyrme model. Phys. Lett. 235B, 147 (1990)

17. Kugler, M., Shtrikman, S.: A new Skyrmion crystal. Phys. Lett. 208B, 491 (1988)

Castillejo, L., Jones, P.S.J., Jackson, A.D., Verbaarschot, J.J.M., Jackson, A.: Dense Skyrmion systems. Nucl. Phys. A501, 801 (1989)

18. Atiyah, M.F.: Geometry of Yang-Mills fields. Lezioni Fermiane. Pisa: Scuola Normale Superiore, 1979

19. Schwartz, A.: On regular solutions of euclidean Yang-Mills equations. Phys. Lett. 67B, 172 (1977)

Jackiw, R., Rebbi, C.: Degrees of freedom in pseudoparticle systems. Phys. Lett. 67B, 189 (1977)

Brown, L., Carlitz, R., Lee, C.: Massless excitations in pseudoparticle fields. Phys. Rev. D16, 417 (1977)

Atiyah, M.F., Hitchin, N., Singer, I.: Deformations of instantons. Proc. Natl. Acad. Sci. USA 74, 2662 (1977)

20. 't Hooft, G.: Unpublished

Corrigan, E., Fairlie, D.B.: Scalar field theory and exact solutions to a classical $S U(2)$ gauge theory. Phys. Lett. 67B, 69 (1977)

21. Jackiw, R., Nohl, C., Rebbi, C.: Conformal properties of pseudoparticle configurations. Phys. Rev. D15, 1642 (1977)

22. Hartshorne, R.: Stable vector bundles and instantons. Commun. Math. Phys. 59, 1 (1978)

23. Berger, M.: Geometry 2. Berlin, Heidelberg, New York: Springer 1987 
24. Verbaarschot, J.J.M., Walhout, T.S., Wambach, J., Wyld, H.W.: Symmetry and quantization of the two-Skyrmion system: the case of the deuteron. Nucl. Phys. A468, 520 (1987)

25. Walhout, T.S.: Multiskyrmions as nuclei. Nucl. Phys. A531, 596 (1991)

26. Hosaka, A., Griffies, S.M., Oka, M., Amado, R.D.: Two Skyrmion interaction for the AtiyahManton ansatz. Phys. Lett. 251B, 1 (1990)

Hosaka, A., Oka, M., Amado, R.D.: Skyrmions and their interactions using the Atiyah-Manton construction. Nucl. Phys. A530, 507 (1991)

27. Crutchfield, W.Y., Snyderman, N., Brown, V.R.: The deuteron in the Skyrme model. Phys. Rev. Lett. 68, 1660 (1992)

28. Massey, W.S.: The quotient space of the complex projective plane under conjugation is a 4sphere. Geom. Dedicata 2, 371 (1973)

Kuiper, N.H.: The quotient space of $\mathbb{C} P_{2}$ by complex conjugation is the 4-sphere. Math. Ann. 208, 175 (1974)

29. Sommerville, D.M.Y.: Non-Euclidean geometry (Chap. 9). London: Bell 1914

Communicated by A. Jaffe 